\title{
Quantile Multi-Armed Bandits: Optimal Best-Arm Identification and a Differentially Private Scheme
}

\author{
Konstantinos E. Nikolakakis ${ }^{\dagger}$, Dionysios S. Kalogerias ${ }^{\dagger}$, Or Sheffet**, Anand D. Sarwate*
}

\begin{abstract}
We study the best-arm identification problem in multi-armed bandits with stochastic rewards when the goal is to identify the arm with the highest quantile at a fixed, prescribed level. First, we propose a successive elimination algorithm for strictly optimal best-arm identification, show that it is $\delta$-PAC and characterize its sample complexity. Further, we provide a lower bound on the expected number of pulls, showing that the proposed algorithm is essentially optimal up to logarithmic factors. Both upper and lower complexity bounds depend on a special definition of the associated suboptimality gap, designed in particular for the quantile bandit problem - as we show, when the gap approaches zero, best-arm identification is impossible. Second, motivated by applications where the rewards are private information, we provide a differentially private successive elimination algorithm whose sample complexity is finite even for distributions with infinite support and characterize its sample complexity. Our algorithms do not require prior knowledge of either the suboptimality gap or other statistical information related to the bandit problem at hand.
\end{abstract}

Index Terms-Quantile Bandits, Best-Arm Identification, Value at Risk, Differential Privacy, Sequential Estimation

\section{INTRODUCTION}

$\mathbf{M}$ ULTI-armed bandits are an important class of online learning problems with a rich history (see the book by [1] for a detailed treatment). In a stochastic $K$-armed bandit problem, a learner is presented with a set of $K$ different actions (or arms) $\{1,2, \ldots, K\}$ and can sequentially take actions (pull arms) to receive random rewards. The reward of arm $i$ at time $t$ is $X_{t}^{i}$. The learner may have one of a number of common objectives, such as to find the arm with the maximum $\mu_{i}=$ $\mathbb{E}\left[X^{i}\right]$ to minimize cumulative regret [2, 3].

In this paper, we study a different form of bandit problems in which the figure of merit is the left-side q-quantile of the involved reward distributions, defined, for arm $i$, as $F_{i}^{-1}(q)=\inf \left\{x: F_{i}(x) \geq q\right\}$, where $F_{i}(x)=\mathbb{P}\left[X^{i} \leq x\right]$ is the corresponding cumulative distribution function (CDF) [4, 5]. In particular, we study the problem of best-arm identification, i.e., that of identifying the arm with the highest or lowest $q$-quantile, with as few samples as possible.

The quantile bandit problem arises naturally in the context of risk-aware optimization and learning, which has expanded

${ }^{\dagger}$ K.E. Nikolakakis and D.S. Kalogerias are with the Department of Electrical Engineering, Yale University, New Haven, CT, 06511 USA, (email: \{konstantinos.nikolakakis, dionysis.kalogerias\}@yale.edu).

*A.D. Sarwate is with the Department of Electrical and Computer Engineering, Rutgers, The State University of New Jersey, Piscataway, NJ, 08854 USA (e-mail: anand.sarwate@ rutgers.edu).

** Or Sheffet is with the Faculty of Engineering, Bar-Ilan University, Ramat Gan, 5290002, Israel (e-mail: or.sheffet@biu.ac.il). considerably during the last decade [6]-[19]. There are many application scenarios which fit this quantile-based risk-aware setting:

1) Arms are different feasible asset portfolio allocations [20] and the goal is to find the portfolio with the minimum potential monetary loss, within a target investment risk $q$. If such a (random) loss is denoted by $Z$, then this goal may be achieved by choosing $F_{Z}^{-1}(1-q)$ as the corresponding objective (to be minimized). In this context, the $(1-q)$-quantile is well-known as the Value-at-Risk at level $q$, denoted as $\mathrm{V}_{0} @ \mathrm{R}_{q}(Z) \equiv F_{Z}^{-1}(1-q)$.

2) Arms are different servers which can be assigned jobs and the rewards are delays. The goal is to identify the server with the highest 95th percentile delay because "waiting for the slowest $5 \%$ of the requests to complete is responsible for half of the total 99\%-percentile latency" [21].

3) Arms are different strains of an illness (e.g. different lung cancer genotypes) and the rewards are effectiveness of a proposed treatment on the strain. We wish to find the strain for which the treatment guarantees the highest effectiveness in at least $90 \%$ of patients.

From a technical standpoint, the quantile bandit problem differs from the mean (or risk-neutral) bandit problem in a number of important ways. First, for the mean, the suboptimality gap between the optimal $i^{*}$ and a suboptimal arm $i$ is simply $\mu_{i^{*}}-\mu_{i}$, whereas the absolute difference between quantiles is less useful. In fact, we show that the difference between the quantile of the optimal and a suboptimal arm can be arbitrarily small, while the hardness of best-quantile-arm identification remains insensitive. As we discuss below, the latter is captured by our gap definition for quantile bandits (Section IV-B], which shows that the difficulty of the problem depends on the levels of the CDF in the neighborhood of the quantile rather than the actual values of quantile. Further, in contrast with the risk-neutral problem, the complexity of the quantile bandit problem is not affected by the tails or the range of the distributions' domain (support). Our analysis elucidates the above properties and provides guarantees on the proposed $\delta$-PAC (probably approximately correct) algorithm for highest quantile identification. In fact, we show that our new gap definition yields a fundamental quantity for the bestquantile identification problem, and the algorithm that we propose returns strictly optimal solutions.

The advent of wide-scale data analytics has made privacy issues a growing concern. Differential privacy (DP) [22] has become the de-facto gold standard for privacy preserving dataanalysis. For quantile bandit problems involving the data of 
individuals it is natural to model the reward information as private or sensitive. For example, the outcome of a treatment on an individual may be private information, but we would still like to find the most effective treatment. We therefore like to both identify the arm with the best quantile and protect the privacy of individuals. The goal is to minimize the "cost of privacy": how many more samples does the private algorithm need over the non-private algorithm? To understand this, it is necessary to have a non-private baseline to measure against; our characterization of the sample complexity of the non-private problem establishes such a baseline. We thus dedicate the first part of this work to showing that our notion of gap fully characterizes solvable instances.

More specifically, in this paper we make the following contributions:

- We provide a novel concentration bound for quantile estimates from $n$ i.i.d. samples, that applies for both continuous and discrete distributions. This result holds for any choice of $n$, thus it is useful for any generic sequential estimation procedure. Additionally, it efficiently captures the effect of quantile level values (close to zero $q \rightarrow 0$ and close to one $q \rightarrow 1$ ) and provides meaningful bounds for any $q \in(0,1)$, in contrast with alternative results in prior works that are uniform over $q$. Our concentration bound allows to solve the problem exactly and indicates that $\epsilon$-approximation bounds that appear in prior works are unnecessary.

- We provide a definition of the gap $\Delta_{i}$ at level $q$ between arm $i$ and the optimal arm that generalizes those proposed in prior work $[5,23,24,1$, Our gap precisely captures the difficulty of the problem in the sense that when $\Delta_{i}=0$ for all suboptimal arms $i$, no algorithm can hope to identify the arm with the higher $q$-quantile (Theorem 2). The latter shows that our definition of gap provides a fundamental quantity for the best-quantile identification problem.

- We introduce a new pure-exploration successive elimination algorithm for quantile bandits (Algorithm 1), show that it is $\delta$-PAC (Theorem 3) and provide nearly matching upper (Theorem 4) and lower (Theorem 5) bounds on the sample complexity that depend on our improved gap definition. In fact, the upper bound (Theorem 4 ) on the termination time of the algorithm is a high probability result, while the converse is a lower bound on the expectation of the termination time (Theorem 5). These results complement prior work on $\varepsilon$-optimal quantile bandits ${ }^{2}[5,23,24$ ] with $\delta$-PAC upper bounds and expectation lower bounds (as we explain later, the difference in the type of upper and lower bounds is probably intrinsic to the problem setting under consideration). Our approach provides optimal solutions, and exact best arm identification for both continuous and discontinuous distributions in contrast with the $\varepsilon$-approximations of prior works, for which the algorithm does not terminate at levels of discontinuities when $\varepsilon \rightarrow 0$. Additionally, the approach of this work does not require any prior knowledge of either

\footnotetext{
${ }^{1}$ A simultaneous (April 2021) preprint 24 considers a similar gap as ours using the lower quantile function (Ramdas, personal communication, 2020).

${ }^{2}$ An arm $i \in \mathcal{A}$ is $\varepsilon$-optimal at a level $q$ if and only if $F_{i}^{-1}(q+\varepsilon) \geq$ $F_{i^{*}}^{-1}(q)$. For the definition and examples of the $\varepsilon$-optimality at a level $q$ see also [5] Definition 2].
}

the suboptimality gap or other statistical information related to the bandit problem at hand. On the other hand, for $\varepsilon$ approximations to achieve the best approximation the value of $\varepsilon$ has to be chosen smaller than the value of gap, which is not known beforehand.

- Using our modified confidence intervals, we propose the first differentially private best-arm identification algorithm for quantile bandits (Algorithm 2), prove that it is private (Theorem 6), and analyze the trade-off between privacy budget and sample complexity (Theorems 7 and 8 . Interestingly, the sample complexity bound for our private algorithm has no dependency on the support size of the distribution, which is necessary in the case where one wishes to privately estimate the $q$-quantile [25]-[27] rather than identify which arm has highest quantile. This difference between estimation and identification may be of interest for future private algorithms.

\section{A. Prior Work}

Most works on bandit problems under stochastic rewards considers the problem of best-arm identification for the mean. This setting received renewed attention after the work of EvenDar, Mannor, and Mansour [28] on the MAB problem in the PAC learning setting. Later work follows by considering extensions/variations of this problem [29]-[33]. Lower bounds on the sample complexity in terms of the mean suboptimiality gap were proved by Mannor and Tsitsiklis [34], and Anthony and Bartlett [35]. Alternative lower bounds also include results based on the KL-divergence of the arms' distributions [36]-[39]. Cappé et al. [40] present the KL-UCB algorithm that achieves (asymptotically) optimal sample complexity rates by matching known lower bounds. In parallel, prior works encompass nonstochastic approaches [41, 42], as well.

Bandit models with non-stationary [43, 44], or heavytailed [45, 46] distributions are most related to this work, since the quantile problem is often of interest in these settings. Kagrecha et al. [16] consider the unbounded reward best-arm identification problem while variants of regret-based approaches include minimization of generalized loss functions [47]-[50]. More recent works also consider risk measures, for instance conditional value-at-risk (CVaR) [4, 17], mean-variance [7, 51. 52] or unified approaches [53]. These are complemented by concentration results on risk measure estimators [54]-[56].

Our results are closely related to prior work on quantile bandit problem for best-arm identification [4, 5, 23, 24, 57]. Altschuler et al. [58] specifically study median identification for contaminated distributions in the robust statistics sense. Of these, the most highly related works are the beautiful work by Szörényi et al. [5], the refinement by David and Shimkin [23], and the preprint of Howard and Ramdas [24]. Our algorithm uses successive elimination (similarly to [5]), while Howard and Ramdas [24] consider the UCB approach. David and Shimkin [23] and Howard and Ramdas [24] tighten the upper bounds to a double-logarithmic factor. The epoch-based algorithms provide asymptotically tighter sample complexity bounds at the expense of a much larger constant. We therefore present both versions of the successive elimination algorithm for best quantile identification; the standard and the epochbased approach. Our results for the quantile bandits problem 
complement the prior works by Szörényi et al. [5] and David and Shimkin [23] by solving the problem of exact arm identification, rather than providing an approximation. A discussion about exact and approximate approaches follows.

1) Comparison with $\varepsilon$-approximate approaches: The aforementioned works [5, 23] study $\varepsilon$-approximate best-arm identification: the algorithm returns an arm which is within $\varepsilon$ of optimal, for some $\varepsilon \geq 0$. First we discuss the major differences on the approach, algorithm and theoretical guarantees in this work and those in prior works. Then we continue by stating advantages and disadvantages between our approach and $\varepsilon$-approximations. To begin with, the neat algorithm and analysis by Szörényi et al. [5] solves the problem of quantile bandits in a variety of cases. These cases include continuous and discrete distributions for $\varepsilon>0$ and continuous distributions for $\varepsilon=0$. That is, the algorithm by Szörényi et al. [5] does not terminate in case of discontinuous distribution for $\varepsilon=0$ when we are interested at the level of discontinuity. This fact can be verified theoretically and experimentally. Theorem 1 by Szörényi et al. [5] (for $\varepsilon=0$ ) involves a gap whose definition is slightly different than our gap. In many cases of discrete distributions the gap by Szörényi et al. [5] is zero, while the gap in this work is positive, showing that the problem instance is not hard. Thus the algorithm of this paper terminates at levels of quantiles with discontinuity as long as the problem is feasible.

To understand this further, we explain a key difference between the algorithm by Szörényi et al. [5] and the algorithm of this work. As we discuss below, this difference is also crucial for the performance of the two algorithms. The decision rule in Algorithm 1 (lines 9 and 11) by Szörényi et al. [5] involves different statistics than those that we consider. Specifically, to characterize the setting of $\varepsilon=0$ for discontinuous distributions both $\hat{Q}_{t, X^{i}} \triangleq \inf \left\{\xi: \hat{\mathbb{P}}_{t}\left[X^{i} \leq \xi\right] \geq q\right\}$ and $\hat{Q}_{t, X^{i}}^{-} \triangleq \inf \{\xi$ : $\left.\hat{\mathbb{P}}_{t}\left[X^{i} \leq \xi\right]>q\right\}$ are required (see Algorithm 1 line 13), while their work [5, Algorithm 1] involves only the quantity $\hat{Q}_{t, X^{i}}$. That difference together with the "less or equal than" (current work) instead of a strict inequality (prior work [5]) in the elimination step, are sufficient to make the algorithm terminate for cases of $\varepsilon=0$ and discontinuous distributions. As a consequence of the different statistics involved, the proof of the concentration bound is also different. Our approach is based on Hoeffding's inequality, while the proof by Szörényi et al. [5] considers Massart's DKW inequality. Notice that the latter of the two approaches does not directly provide a concentration bound for the statistic $\hat{Q}_{t, X^{i}}^{-}$, however the Chernoff-Hoeffding bound solves the problem in the expense of a larger constant. For an alternative approach of the concentration bound proof that involves smaller constants and uniformity over all quantiles see also the preprint by Howard and Ramdas [24].

Szörényi et al. [5] use a gap which depends on the parameter $\varepsilon$, while other prior works [23, 24] provide an alternative gap that does not involve the quantity $\varepsilon$, but study $\varepsilon$-approximate algorithms. By contrast, our algorithm returns the optimal arm, and we show that when our gap is 0 then a suboptimal distribution (with small $q$-quantile) is actually indistinguishable from a distribution with a larger $q$-quantile (see Section IV-B
Theorem 22. The main advantage of approaches that consider $\varepsilon$-approximations [5, 23, 24] is that the algorithm terminates even when the gap is zero (by breaking ties arbitrarily), at the expense of approximating the quantile estimate $(\varepsilon>0)$. Still, in applications we may not always be able to choose $\varepsilon$ to achieve best approximation unless there is side information for the distributions of the data. For instance, if the value of the gap is not known beforehand, we may accidentally choose $\varepsilon$ to be much greater than the gap and the output of the algorithm can possibly crudely approximate the solution of the problem by returning a rather suboptimal arm. In contrast, the algorithms for exact best arm identification of this work do not require prior knowledge of any side information, making them of interest for these applications. Additionally, our results substantially differ from those by David and Shimkin [23]. Specifically, their Theorem 1 considers the case of continuous distributions, while we provide unified analysis for both discrete and continuous distributions. Further results [23, Theorem 2 and Theorem 3] show that the algorithm by David and Shimkin is not guaranteed to terminate (unbounded expected number of samples) when $\varepsilon \rightarrow 0$. In contrast, the current work solves the problem of exact estimation.

Lastly, in our approach the upper ( $\delta$-PAC) bound does not appear to provide an upper bound for the expected number of pulls, and the lower bound on the expected number of pulls does not directly guarantee a lower $\delta$-PAC bound. Specifically, under the low (at most $\delta$ ) probability event, there exist instances for which the problem reduces to that of zero gap problem. If the (unique) optimal arm is mistakenly eliminated under the low probability event, while at least two distributions of the remaining sub-optimal arms are identical, then the algorithm does not terminate, because the gap restricted to the remaining identical arms is zero. In fact, no algorithm can identify the best-quantile arm under the zero gap case (see Section IV-B Theorem 2); however, $\varepsilon$-approximate solutions (with $\varepsilon>0$ ) terminate by breaking ties arbitrarily.

2) Prior work on Differential Privacy.: The field of differentially private machine learning is, by now, too large to summarize here, as the following (non-exhaustive) list of works discussing learning quantiles/threshold-functions attests [25][27, 59]-[63]. For differentially private multi-armed bandit problems for the mean, Mishra and Thakurta [64] were the first to analyze a differentially private (DP) algorithm for multi-armed bandit, building a private variant of the UCB-algorithm [65] using the tree-based algorithm [66, 67]. Shariff and Sheffet [68] have proven that any $\epsilon$-DP algorithm (see Section V] Definition 4) for the (mean) multi-armed bandit problem must pull each suboptimal arm $i$ at least $\Omega\left(\log (T) / \epsilon\left(\mu_{i^{*}}-\mu_{i}\right)\right)$ many times (with $i^{*}$ denoting the optimal arm, of largest mean-reward $\mu_{i^{*}}=\max _{i \in \mathcal{A}} \mu_{i}$ ) which doesn't quite meet the DP-UCB algorithm's upper bound. Most recently Sajed and Sheffet [69] gave a DP version of successive elimination whose regret matches the lower bound [68].

\section{Problem Statement}

We consider a $K$-armed unstructured stochastic bandit $\nu=$ $\left(\nu_{i}: i \in \mathcal{A}\right)$, where $\mathcal{A} \triangleq\{1,2, \ldots, K\}$ is the set of arms and $\nu_{i}$ 
are probability measures. For the $i$-th arm, let $X^{i}$ be a random variable with distribution $\nu_{i}$. We will describe distributions by their cumulative distribution functions (CDFs).

Definition 1. Let $F_{i}(\cdot)$ be the CDF of $X^{i}$ for arm $i$. The $q$-quantile $F_{i}^{-1}(q)$ is defined as

$$
F_{i}^{-1}(q) \triangleq \inf \left\{\xi: \mathbb{P}\left[X^{i} \leq \xi\right] \geq q\right\}
$$

and the best arm is defined as

$$
i^{*} \triangleq \underset{i \in \mathcal{A}}{\arg \max } F_{i}^{-1}(q) .
$$

For simplicity, we assume that the best arm is unique in the set $\mathcal{A}$. We denote the set of suboptimal arms as $\mathcal{A}^{-i^{*}} \triangleq$ $\{1,2, \ldots, K\} \backslash\left\{i^{*}\right\}$. Given $n$ samples the estimated CDF of $X^{i}$ is $\hat{F}_{n, i}(x) \triangleq \frac{1}{n} \sum_{\ell=1}^{n} \mathbb{I}\left\{X_{\ell}^{i} \leq x\right\}$. We denote the set of samples from arm $k$ as $\left\{X_{i}^{k}\right\}_{i=1}^{n}$, while for the $j^{\text {th }}$ order statistic of $\left\{X_{i}^{k}\right\}_{i=1}^{n}$ we use the standard notation $X_{(j)}^{k}$ for $j \in\{1,2, \ldots n\}$. If the value $j$ in $X_{(j)}^{k}$ appears out of the range $(1, n)$, while $n$ samples are available, then it is considered equal to the closest of the two values 1 or $n$.

An algorithm for our quantile bandit chooses at each time $n$ an arm $i_{n} \in \mathcal{A}$ and obtains a reward $X_{n}^{i_{n}} \sim \nu_{i_{n}}$. The algorithm terminates by stopping sampling and declaring an arm $\hat{k}$ as the arm with the highest $q$-quantile, and succeeds if actually $\hat{k}=i^{*}$. We call an algorithm $\delta$-PAC if $\mathbb{P}\left(\hat{k}=i^{*}\right) \geq 1-\delta$.

\section{CONCENTRATION BOUnd}

We proceed by providing a concentration bound for quantile estimation that applies for both discrete and continuous distributions.

Theorem 1 (Concentration Bound). Choose a level $q \in(0,1)$ Fix $\delta \in(0,1)$. For any $n \in \mathbb{N}$, if

$$
\sqrt{\frac{\log (2 / \delta)}{2 n}} \leq \zeta \leq \min \{q, 1-q\}
$$

then

$$
\mathbb{P}\left(F_{X}^{-1}(q) \notin\left[X_{(\lfloor n(q-\zeta)\rfloor)}, X_{(\lceil n(q+\zeta)\rceil)]}\right) \leq \delta .\right.
$$

In contrast, with concentration bounds that are uniform over the values of the level $q$ [5], Theorem (1) shows the dependence of the required number of samples $n$ with respect to $q$ through the inequality (3). This property explicitly expresses the difficulty of the problem when estimating the quantile close to the tails of the distribution. We provide the proof of Theorem 11 in Appendix B

\section{OPTIMAL BEST-ARM IDENTIFICATION FOR QUANTILE BANDITS}

\section{A. (Non-Private) Successive Elimination Algorithm}

We choose to study successive elimination (SE) rather than a variant of UCB [65] (adopted by Howard and Ramdas [24] for quantiles) for the following reasons. Firstly, we prove matching upper and lower bounds on the sample complexity, showing our SE algorithm is essentially optimal (up to logarithmic terms). Secondly, since we are also interested in developing differentially private algorithms (see Section $\mathrm{V}$ ), the SE algorithm is more "privacy friendly", because the sampling strategy is independent of the data and it uses confidence bounds in terms of the order statistics. Finally, there is no private analog to UCB when the distributions have infinite support.

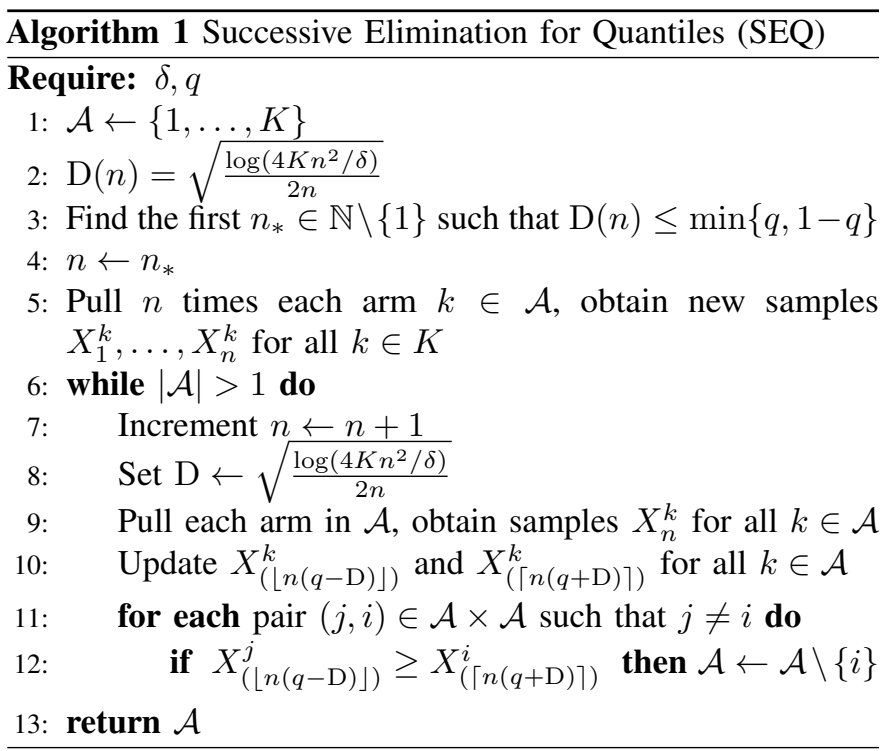

Our Successive Elimination algorithm for Quantiles (SEQ) Algorithm is shown in Algorithm 1. To explain SEQ (Algorithm 1 , we define the sequence $\mathrm{D}(n) \triangleq \sqrt{\log \left(4 K n^{2} / \delta\right) / 2 n}$, (we denote $\mathrm{D}(n)$ as $\mathrm{D}$ for sake of space) and we use a concentration bound on the quantile (see Lemma 1 )

$\mathbb{P}\left(F_{i}^{-1}(q) \in\left[X_{(\lfloor n(q-\mathrm{D})\rfloor)}^{i}, X_{(\lceil n(q+\mathrm{D})\rceil)}^{i}\right]\right)>1-\frac{\delta}{2 K n^{2}}$.

The latter yields the elimination condition in line 13 of Algorithm 1 Specifically, when the inequality $X_{(\lfloor n(q-\mathrm{D})\rfloor)}^{j} \geq$ $X_{(\lceil n(q+\mathrm{D})\rceil)}^{i}$ holds then $F_{i}^{-1}(q) \leq F_{j}^{-1}(q)$ with probability at least $1-\delta$ (by applying union bound in (5) over all times $n$ and arms $K)$. Thus to identify the arm with the maximum quantile, whenever $X_{(\lfloor n(q-\mathrm{D})\rfloor)}^{j} \geq X_{(\lceil n(q+\mathrm{D})\rceil)}^{i}$, we remove $i$ from $\mathcal{A} 3^{3}$

A variant of the algorithm would be to take samples in epochs of increasing size. We consider this approach in the development of the differentially private version of Algorithm 1. which reduces to a non-private epoch-based variant of Algorithm 1] (Section V] Algorithm 2). This epoch-based algorithm improves the logarithmic (and inconsequential) part of the bound of Theorem 4 from $\log \left(1 / \Delta_{i}\right)$ to $\log \log \left(1 / \Delta_{i}\right)$ and matches asymptotically the bound for UCB [24] (see the discussion at the end of Section $\mathrm{V}$.

\section{B. Suboptimality gap}

We first define the suboptimality gap between the best arm $i^{*}$ and any suboptimal arm.

\footnotetext{
${ }^{3}$ To identify the arm with the minimum quantile, we modify line 13 of the algorithm as follows: If $X_{(\lfloor n(q-\mathrm{D})\rfloor)}^{j} \geq X_{(\lceil n(q+\mathrm{D})\rceil)}^{i}$, then we remove $j$ from $\mathcal{A}$.
} 
Definition 2. The suboptimality gap $\Delta_{i}$ (also denoted as $\Delta\left(F_{i}, F_{i^{*}}\right)$ ) between the optimal arm $i^{*}$ and any suboptimal arm $i$ at level $q \in(0,1)$ is

$$
\Delta_{i} \triangleq \sup \left\{\eta \geq 0: F_{i}^{-1}(q+\eta) \leq F_{i^{*}}^{-1}(q-\eta)\right\} .
$$

How can we interpret this gap? Roughly speaking, it is the amount of probability mass needed to swap the order of the quantiles. To get further insight into the definition (6), notice that $\mathrm{D}(n)$ is decreasing with respect to $n$, and the elimination occurs at the first time (maximum value of $\mathrm{D}$ ) that gives $X_{(\lfloor n(q-\mathrm{D})\rfloor)}^{j} \geq X_{([n(q+\mathrm{D})\rceil)}^{i}$. In fact, the value $\Delta_{i}$ in $(6)$ acts as a threshold on the quantity $\mathrm{D}$ in the analysis of the algorithm (proof of Theorem 4). Our definition of gap applies on continuous, discrete, and mixture distributions.

Most importantly, the key point in the Definition 2 of the quantile suboptimality-gap is that it fully characterizes the pairs of distributions for which we can discern that one has a higher $q$-quantile than another from any number of samples. Formally, for a pair of distributions $\left(F_{\mathrm{l}}, F_{\mathrm{h}}\right)$ where the former has a suboptimal $q$-quantile than the latter, namely $F_{1}^{-1}(q) \leq$ $F_{\mathrm{h}}^{-1}(q)$, we define the distance to quantile-flip at $q$ as

$$
\begin{aligned}
& d_{\text {flip }}\left(F_{\mathrm{l}}, F_{\mathrm{h}}\right) \\
& \triangleq \inf _{\left(G_{\mathrm{h}}, G_{1}\right): G_{\mathrm{h}}^{-1}(q)>G_{1}^{-1}(q)} \max \left\{d_{\mathrm{TV}}\left(F_{1}, G_{\mathrm{h}}\right), d_{\mathrm{TV}}\left(F_{\mathrm{h}}, G_{\mathrm{l}}\right)\right\}
\end{aligned}
$$

and by $d_{\mathrm{TV}}(\cdot, \cdot)$ we denote the total variation. Next we provide a rigorous result which shows that the gap $\Delta_{i}$ is indeed a fundamental quantity which characterizes the complexity of the best quantile identification problem.

Theorem 2. For any $0<q<1$ and any two distributions $F_{i}$ and $F_{i^{*}}$ such that $F_{i}^{-1}(q) \leq F_{i^{*}}^{-1}(q)$ it holds that $d_{\text {flip }}\left(F_{i}, F_{i^{*}}\right)=\Delta\left(F_{i}, F_{i^{*}}\right)$ provided that $\Delta\left(F_{i}, F_{i^{*}}\right)<$ $\min \{q, 1-q\} / 2$.

We provide the proof of Theorem 2 in Appendix $\mathrm{A}$ Theorem 2 shows that if $\Delta_{i}=0$ then $d_{\text {flip }}\left(F_{1}, F_{\mathrm{h}}\right)=0$ and no algorithm can distinguish which arm has the higher $q$-quantile, regardless of its sample-size: every batch of samples can be generated by a quantile flip pair $\left(G_{\mathrm{h}}, G_{1}\right)$ with the same probability. Conversely, when $\Delta_{i}>0$ we devise an algorithm that discerns which arm has the higher $q$-quantile using $\tilde{O}\left(\Delta_{i}^{-2}\right)$ many example ${ }^{4}$ from each arm and argue that this bound is optimal in the sense that there exists a collection of $K$ distributions requiring $\tilde{O}\left(\Delta_{i}^{-2}\right)$ many examples from each distribution (Section IV-C). Corollary 1 in Appendix C provides the cases for which $\Delta>0$ or $\Delta=0$. We continue by providing graphical representations and properties of the gap in certain cases. Finally, we present the main differences between our definition and definitions in prior work.

1) Graphical illustration of the gap: Figure 1 shows the gap as a function of the level $q$ of the quantile for two continuous distributions, the Gaussian and the exponential. We vary the optimal distribution by altering the parameter (variance or rate). For the Gaussian example (left) we look at the gap between a (suboptimal) $\mathcal{N}(0,2)$ distribution and Gaussians of higher variance. As expected, when looking at the median the gap is 0

\footnotetext{
${ }^{4}$ The notation $\tilde{O}(\cdot)$ denotes order up to logarithmic factors.
}
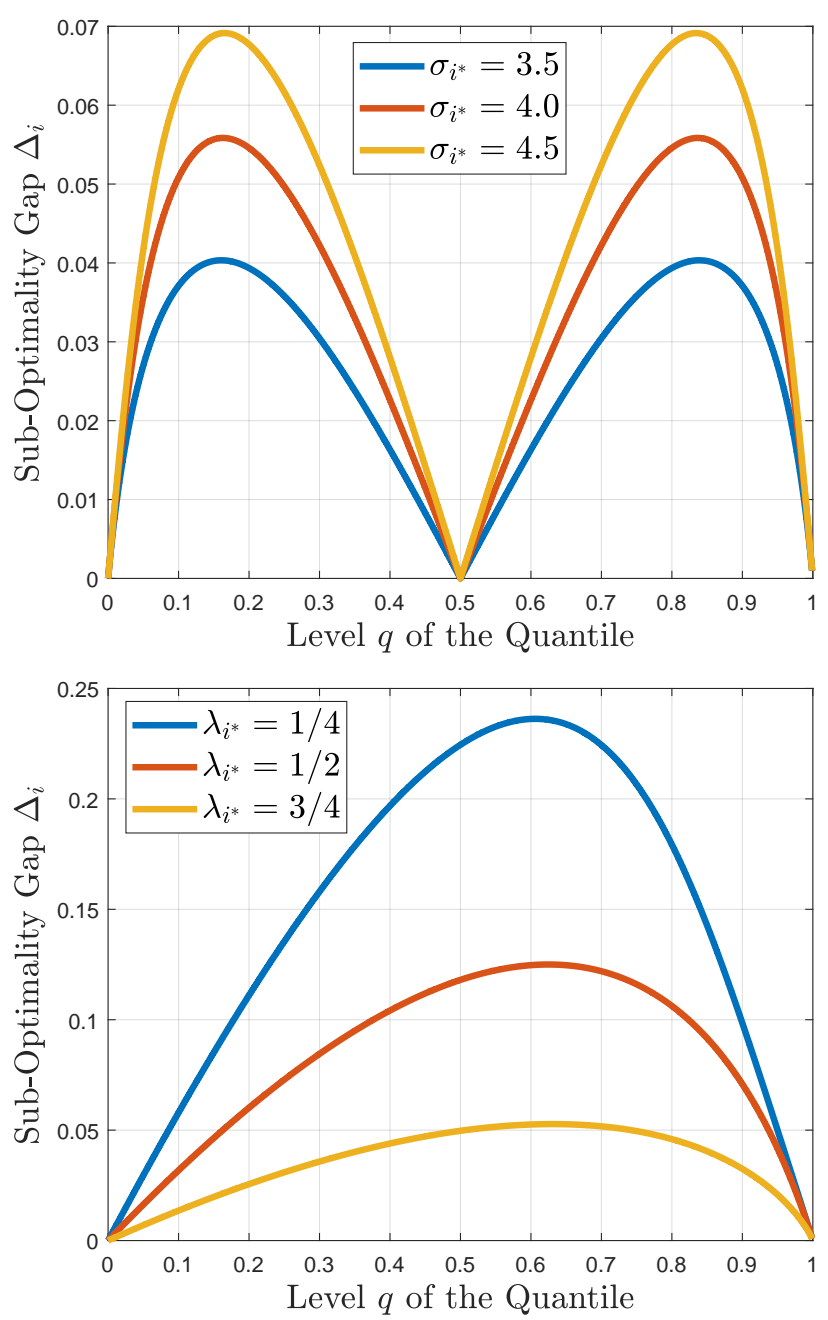

Fig. 1. Illustration of the suboptimality gap for two example distributions. Upper: Gaussian with $\mu_{i^{*}}=\mu_{i}=0, \sigma_{i}=2$ and different values of $\sigma_{i^{*}}$, Lower: Exponential with $\lambda_{i}=1$.

since they are both symmetric distributions. More interestingly, the best-arm identification problem becomes easiest when looking at some quantile $q^{*}$ (or $1-q^{*}$ ) that lies between $1 / 2$ and 1 . The problem becomes hard again when looking at the tails of the distribution. For the exponential distribution we compare to a rate $\lambda_{i}=1$ for smaller values of the rate. As the difference in rates grows, the problem becomes easier, as expected. Here too we see an optimal $q^{*}$ between $1 / 2$ and 1 for which the top quantile is easiest to identify. While analytical expressions for these optimal points could possibly be derived through analyzing the corresponding densities, this is not the focus of our work.

For discrete distributions, we can show that the difference between the quantiles can become arbitrarily small while the definition of the gap and the sample complexity of Algorithm 1 remain insensitive, see Figure 2 . Additionally, while the difference between the quantile values is not the correct definition to use for the gap in general, the two quantities are related in the case of Lipschitz CDFs.

Proposition 1. Suppose $F$ and $G$ are two distributions with L-Lipschitz continuous and strictly increasing CDFs. Then the 
following inequality holds $\Delta(F, G) \leq \frac{L}{2}\left|F^{-1}(q)-G^{-1}(q)\right|$.

Proof of Proposition 1 By definition, we have

$$
\begin{aligned}
\eta & =\left|F\left(F^{-1}(q+\eta)\right)-F\left(F^{-1}(q)\right)\right| \\
& \leq L\left|F^{-1}(q+\eta)-F^{-1}(q)\right|=L\left(F^{-1}(q+\eta)-F^{-1}(q)\right) \\
\eta & =\left|G\left(G^{-1}(q)\right)-G\left(, G^{-1}(q-\eta)\right)\right| \\
& \leq L\left|G^{-1}(q)-G^{-1}(q-\eta)\right|=L\left(G^{-1}(q)-G^{-1}(q-\eta)\right) .
\end{aligned}
$$

So,

$2 \eta+L\left(G^{-1}(q-\eta)-F^{-1}(q+\eta)\right) \leq L\left(G^{-1}(q)-F^{-1}(q)\right)$.

From the definition of the gap, taking the supremum over $\eta$ gives

$$
\Delta(F, G) \leq \frac{L}{2}\left|F^{-1}(q)-G^{-1}(q)\right| .
$$

This completes the proof.

We continue by providing the difference between our definition for the gap in comparison with a similar definition in prior work. By providing a simple example, we explain that previous definitions fail to capture certain cases of interest.

2) Difference between the proposed gap and prior work: Although the suboptimality gap we propose (Definition 2) may look similar to those proposed in prior works [5, 23], there are several important differences. Earlier work by Szörényi et al. [5] explicitly incorporates the approximation parameter, whereas our definition depends only on the arm distributions. Further, the proposed gap differs from that in [5] when $\varepsilon=0$, because "less or equal than" takes the place of a strict inequality. This is not a trivial point, because for the case of discrete distributions the gap by Szörényi et al. can be zero, while the gap of the present work is positive, Algorithm 1 terminates and the problem is not hard. For instance, consider an example of two arms with the sub-optimal arm following a Bernoulli distribution with probability $1 / 2$ and support $\{1,2\}$, and with the optimal arm taking the value 2 with probability 1 . Then the problem is not hard in terms of the sample complexity; Definition 2 gives $\Delta_{i}>0$, while the gap in Szörényi et al. [5] is zero when $\varepsilon=0$. Additionally, in Theorem 2 we show that if $\Delta_{i}=0$, no algorithm can identify the best quantile-arm with probability greater than $1 / 2$. Further, we provide a minimax lower bound on the expected number of pulls based on the gap $\Delta_{i}$ (Theorem 55; the latter shows that our upper bound is optimal up to logarithmic factors. Finally, the gap in [23] involves a strict inequality instead of "less or equal than" and the supremum involves the distribution of the sub-optimal arm. This definition does not capture the difficulty of the problem for general cases of discrete distributions as we discussed above. The gap in this work (see Definition 2) captures the difficulty of the problem for discrete, continuous distributions, as well as for mixtures.

\section{Analysis}

Our first result guarantees that Algorithm 1 eliminates the suboptimal arms while the unique best arm remains in the set $\mathcal{A}$ with high probability until the algorithm terminates.
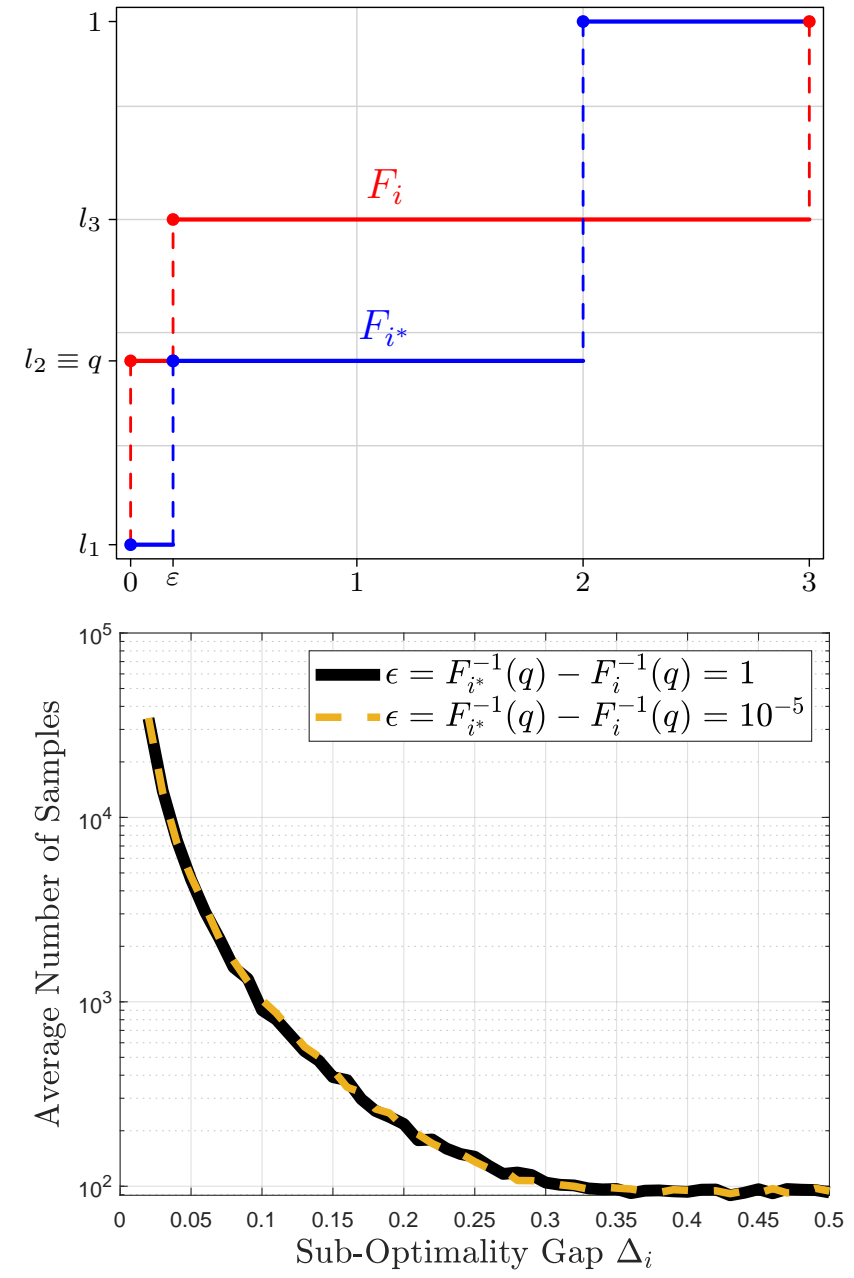

Fig. 2. Upper: Distributions $F_{i^{*}}(\cdot), F_{i}(\cdot)$ of the optimal and suboptimal arm. Note that $0=F_{i}^{-1}(q)<F_{i^{*}}^{-1}(q)=\epsilon$, for some $\epsilon \in(0,1]$. However, the gap is $\Delta_{i}=\min \left\{l_{3}-q, q-l_{1}\right\}$, independent of $\epsilon$. Lower: Experimental evaluation of the average number of samples at termination of Algorithm 1 for $\epsilon=1$ and $\epsilon=10^{-5}$.

For the rest of the paper we assume that $\Delta_{i}>0$ for all $i \in\{1,2 \ldots, K\} \backslash i^{*}$.

Theorem 3. Algorithm 1$]$ is $\delta$-PAC.

To prove Theorem 3 recall that $n^{*}$ is the smallest integer that satisfies the inequality $\mathrm{D}\left(n^{*}\right) \leq \min \{q, 1-q\}$. First, we show that the event $\mathcal{E}$ defined as

$$
\mathcal{E} \triangleq \bigcap_{k=1}^{K} \bigcap_{n=n^{*}}^{\infty}\left\{F_{k}^{-1}(q) \in\left[X_{(\lfloor n(q-\mathrm{D}(n))\rfloor)}^{k}, X_{(\lceil n(q+\mathrm{D}(n))])}^{k}\right]\right\}
$$

occurs with probability at least $1-\delta$.

Lemma 1. Choose a level $q \in(0,1)$ and fix $\delta \in(0,1)$. Then $\mathbb{P}\left(\mathcal{E}^{c}\right) \leq \delta$.

Proof. For every $n$ such that

$$
\mathrm{D}(n)=\sqrt{\frac{\log \left(4 K n^{2} / \delta\right)}{2 n}} \leq \min \{q, 1-q\},
$$

from Lemma 1 in Appendix B, we get

$$
\mathbb{P}\left(\left\{F_{k}^{-1}(q) \notin\left[X_{(\lfloor n(q-\mathrm{D}(n))\rfloor)}^{k}, X_{(\lceil n(q+\mathrm{D}(n)))]}^{k}\right]\right\}\right)
$$




$$
\leq \frac{\delta}{2 K n^{2}}
$$

and $k \in\{1,2, \ldots, K\}$. We conclude that

$$
\begin{aligned}
& \mathbb{P}\left(\mathcal{E}^{c}\right) \\
& \leq \sum_{k=1}^{K} \sum_{n=n^{*}}^{\infty} \mathbb{P}\left(F_{k}^{-1}(q) \notin\left[X_{(\lfloor n(q-\mathrm{D}(n))\rfloor)}^{k}, X_{(\lceil n(q+\mathrm{D}(n))])}^{k}\right]\right) \\
& \leq \sum_{k=1}^{K} \sum_{n=n^{*}}^{\infty} \frac{\delta}{2 K n^{2}} \\
& =\sum_{n=n^{*}}^{\infty} \frac{\delta}{2 n^{2}} \\
& \leq \delta \sum_{n=1}^{\infty} \frac{1}{2 n^{2}} \\
& \leq \delta .
\end{aligned}
$$

This proves the result stated in the lemma.

We continue by providing the proof of Theorem 3

Proof of Theorem 3 Lemma 1 gives $\mathbb{P}(\mathcal{E})>1-\delta$. Under the event $\mathcal{E}$ the following inequalities hold

$$
F_{j}^{-1}(q) \geq X_{(\lfloor n(q-\mathrm{D}(n))\rfloor)}^{j} \text { and } F_{i}^{-1}(q) \leq X_{(\lceil n(q+\mathrm{D}(n))\rceil)}^{i} .
$$

Every time that the stopping condition $X_{(\lfloor n(q-\mathrm{D}(n))\rfloor)}^{j} \geq$ $X_{(\lceil n(q+\mathrm{D}(n))\rceil)}^{i}$ occurs we eliminate the arm $i$ and the arm $j$ remains in $\mathcal{A}$. The stopping condition and the inequalities in (11) guarantee that

$$
F_{j}^{-1}(q) \geq F_{i}^{-1}(q)
$$

As a consequence the optimal arm $i^{*}$ is not eliminated and the Algorithm stops when $\mathcal{A}=\left\{i^{*}\right\}$.

The next result bounds the total number of pull at termination with high probability.

Theorem 4. Fix $\delta \in(0,1)$. There exists a constant $C>0$ such that the number of samples $\tau$ (and total number of pulls) of Algorithm 1 satisfies, with probability at least $1-\delta$,

$$
\tau \leq C \sum_{i \in \mathcal{A}^{-i^{*}}} \frac{\log \frac{K}{\delta}+\log \left(\frac{1}{\Delta_{i}}\right)}{\Delta_{i}^{2}}
$$

From the proof of Theorem 4, it also follows that the number of pulls for each suboptimal arm $i \in \mathcal{A}^{-i^{*}}$ is at most $\mathcal{O}\left(\log \left(K / \delta \Delta_{i}\right) / \Delta_{i}^{2}\right)$. The upper bound indicates that the number of pulls (with high probability) is proportional to the quantity $1 / \Delta_{i}^{2}$ up to a logarithmic factor for each suboptimal arm $i$. In fact, experimental results on SEQ (Algorithm 1) show that the explicit bound in (13) matches the average number of pulls in the experiment. Next we provide the proof of Theorem 4. For the simulation results we refer the reader to Section VI Figure 3 .

Proof of Theorem 4 Under the event $\mathcal{E}$, we will find a bound on the smallest value of $n$ that satisfies the inequality
$X_{(\lfloor n(q-\mathrm{D}(n))\rfloor)}^{i^{*}} \geq X_{(\lceil n(q+\mathrm{D}(n))\rceil)}^{i}$ for all $i \neq i^{*}$. When $\mathcal{E}$ occurs, it is true that

$$
\begin{aligned}
X_{(\lfloor n(q-\mathrm{D}(n))\rfloor)}^{i^{*}} & \geq X_{(\lceil n(q-\mathrm{D}(n))-1\rceil)}^{i^{*}} \\
& \stackrel{(\mathrm{A})}{\geq} F_{i^{*}}^{-1}(q-2 \mathrm{D}(n)-1 / n) \\
F_{i}^{-1}(q+2 \mathrm{D}(n)+1 / n) & \stackrel{(\mathrm{B})}{\geq} X_{(\lfloor n(q+\mathrm{D}(n)+1 / n)\rfloor)}^{i} \\
& \geq X_{(\lceil n(q+\mathrm{D}(n))\rceil)}^{i}
\end{aligned}
$$

and (A), (B) come from the definition of $\mathcal{E}$. From the definition of the suboptimality gap follows that $F_{i^{*}}^{-1}\left(q-\Delta_{i^{*}}\right) \geq F_{i}^{-1}(q+$ $\Delta_{i}$ ). The latter together with (14) and (15) give that it is sufficient to find the smallest value of $n$ that satisfies the inequalities

$$
\begin{aligned}
& F_{i^{*}}^{-1}(q-2 \mathrm{D}(n)-1 / n) \geq F_{i^{*}}^{-1}\left(q-\Delta_{i}\right), \\
& F_{i}^{-1}\left(q+\Delta_{i}\right) \geq F_{i}^{-1}(q+2 \mathrm{D}(n)+1 / n) .
\end{aligned}
$$

We denote by $\tau_{i}$ the total number of pulls for a suboptimal arm $i$. The monotonicity of $F_{i^{*}}(\cdot), F_{i}(\cdot)$ and (16, ,17) give

$$
\Delta_{i} \geq 2 \mathrm{D}(n)+\frac{1}{n} \Longrightarrow \Delta_{i} \geq 2 \sqrt{\frac{\log \left(4 K n^{2} / \delta\right)}{2 n}}+1 / n
$$

and the values of $n$ that satisfy the inequality above are bounded by

$$
\tau_{i}=\mathcal{O}\left(\frac{\log \frac{K}{\delta \Delta_{i}}}{\Delta_{i}^{2}}\right) .
$$

To conclude, the total number of samples $\tau$ is $\sum_{i \in \mathcal{A}^{-i^{*}}} \tau_{i}$ with probability at least $1-\delta$.

We next present a lower bound on the expected number of pulls. Szörényi et al. [5] use the results of Mannor and Tsitsiklis [34] to obtain a bound that depends on $\max \left\{\varepsilon, \Delta_{i}\right\}$ (for some chosen $\varepsilon>0$ ). We use the approach suggested in the book of Lattimore and Szepesvári [1] on a different class of distributions and get a bound that depends only on $\Delta_{i}$.

Theorem 5. Fix $\delta \in(0,1)$. There exists a quantile bandit with $K$-arms and gaps $\Delta_{i} \in(0,1 / 4), i \in[K]^{-i^{*}}$, such that

$$
\inf _{\delta-\text { PAC policy }} \mathbb{E}_{\pi}[\tau] \geq \sum_{j=1, j \neq i^{*}}^{K} \frac{3(1-q)^{2}}{100 \Delta_{j}^{2}} \log \left(\frac{1}{4 \delta}\right) .
$$

From Theorem 5, it follows that, up to logarithmic factors depending on $\delta$ (Theorem 5), and also $K, \Delta_{i}, i \in \mathcal{A}^{-i^{*}}$ (Theorem 4), Algorithm [1 is (almost) optimal relative to the expected number of pulls achieved, and its performance is necessarily inversely proportional to the square of our suboptimality gap. More interestingly, our lower bound shows that as $\Delta_{i} \rightarrow 0$ the sample complexity goes to $\infty$ and indeed as Theorem 2 shows, $\Delta_{i}=0$ implies that the best-quantile arm identification problem is impossible.

Proof of Theorem 4. We note that to prove a minimax lower bound we need only show a "bad instance" of the problem. It is convenient for the proof to use a mixed discrete/continuous 
distribution since the calculations are easier. We therefore define the following class of distributions:

$$
g^{w}(x) \triangleq w \delta(x)+(1-w), \quad x \in[0,1],
$$

i.e., a mixture of a mass (Dirac delta) at 0 and a uniform distribution on $[0,1]$. Let $G^{w}$ be the cumulative distribution function of $g^{w}$. The KL-divergence between two such distributions is

$$
\boldsymbol{D}_{\mathrm{KL}}\left(g^{w} \| g^{w^{\prime}}\right)=w \log \frac{w}{w^{\prime}}+(1-w) \log \frac{1-w}{1-w^{\prime}},
$$

which is the same as the divergence between two Bernoulli random variables. The gap between $g^{w}$ and $g^{w+\gamma}$ for $q>w+\gamma$ and small $\gamma<\frac{1}{2}(q-w)$ is $\Theta(\gamma)$. To see this, let $\nu=g^{w}$ and $\nu^{\prime}=g^{w+\gamma}$, so $\nu$ has the higher $q$-quantile. We can calculate the $(q-\eta)$-quantile of $\nu$ and the $(q+\eta)$-quantile of $\nu^{\prime}$ as

$$
\begin{aligned}
x & =\frac{q-\eta-w}{1-w} \\
x^{\prime} & =\frac{q+\eta-(w+\gamma)}{1-(w+\gamma)} .
\end{aligned}
$$

We need to find the inf over all $\eta$ such that $x^{\prime}<x$. By taking the case of equality, we find

$$
\begin{aligned}
\frac{q-\eta-w}{1-w} & =\frac{q+\eta-(w+\gamma)}{1-(w+\gamma)} \Longleftrightarrow \\
(q-\eta-w)(1-w-\gamma) & =(q+\eta-w-\gamma)(1-w) \Longleftrightarrow \\
\gamma(1-q) & =\eta(2-2 w-\gamma) \Longleftrightarrow \\
\eta & =\frac{1-q}{2-2 w-\gamma} \gamma
\end{aligned}
$$

We adapt a strategy for the mean-bandit problem appearing in [1. Section 33.2] to the quantile bandit setting. Let $\mathcal{E}$ denote a class of environments for the bandit problem and $\nu \in \mathcal{E}$ be a particular environment (i.e. setting of the arm distributions). Let $i^{*}(\nu)$ be the optimal arm 5 which we will denote by $i^{*}$ when $\nu$ is clear from context.

Fix $\gamma<1 / 6$. Recall that $G^{w}$ is the CDF of $g^{w}$ given by 21). Let $\nu^{(1)}$ be defined by the arm CDFs

$$
\nu_{i}^{(1)}= \begin{cases}G^{1 / 3-\gamma} & i=1 \\ G^{1 / 3} & i \neq 1 .\end{cases}
$$

The gap between $\nu_{1}^{(1)}$ and $\nu_{i}^{(1)}$ is (setting $w=1 / 3-\gamma$ and using the fact that $\gamma<1 / 6$ ):

$$
\Delta_{i}=\frac{1-q}{2-2 w-\gamma} \gamma=\frac{1-q}{4 / 3+\gamma} \gamma \geq \frac{3}{5}(1-q) \gamma .
$$

For each $j$ define $\nu^{(j)}$

$$
\nu_{i}^{(j)}=\left\{\begin{array}{ll}
G^{1 / 3-\gamma} & i=1 \\
G^{1 / 3-2 \gamma} & i=j \\
G^{1 / 3} & i \neq 1, j
\end{array} .\right.
$$

Let $\pi$ be a $\delta$-PAC policy. Then we have $\mathbb{P}_{\nu^{(1)}}(\hat{k} \neq 1) \leq \delta$ and $\mathbb{P}_{\nu^{(j)}}(\hat{k} \neq j) \leq \delta$. Since $\nu^{(1)}$ and $\nu^{(j)}$ differ in only a single arm distribution, we have [1, Lemma 15.1]

$$
\boldsymbol{D}_{\mathrm{KL}}\left(\mathbb{P}_{\nu^{(1)}} \| \mathbb{P}_{\nu^{(j)}}\right)=\sum_{i=1}^{K} \mathbb{E}_{\nu^{(1)}}\left[T_{i}(n)\right] \boldsymbol{D}_{\mathrm{KL}}\left(P_{\nu_{i}^{(1)}} \| P_{\nu_{i}^{(j)}}\right)
$$

${ }^{5}$ For the example in Theorem 4 there is a unique optimal arm.

$$
=\mathbb{E}_{\nu^{(1)}}\left[T_{j}(\tau)\right] \boldsymbol{D}_{\mathrm{KL}}\left(G^{1 / 3} \| G^{1 / 3-2 \gamma}\right)
$$

and

$$
\begin{aligned}
& \boldsymbol{D}_{\mathrm{KL}}\left(G^{1 / 3} \| G^{1 / 3-2 \gamma}\right) \\
& =\frac{1}{3} \log \frac{1 / 3}{1 / 3-2 \gamma}+\frac{2}{3} \log \frac{2 / 3}{2 / 3+2 \gamma} \\
& =\frac{1}{3} \log \frac{1}{1-6 \gamma}+\frac{2}{3} \log \frac{1}{1+3 \gamma} \\
& \leq \frac{1}{3}\left(6 \gamma+18 \gamma^{2}+54 \gamma^{3}\right)-\frac{2}{3}\left(3 \gamma-\frac{9}{2} \gamma^{2}\right) \\
& =9 \gamma^{2}+18 \gamma^{3},
\end{aligned}
$$

where we used the inequalities $\log \frac{1}{1-x} \leq x+\frac{x^{2}}{2}+\frac{x^{3}}{3}$ and $-\log (1+x) \leq-x+\frac{x^{2}}{2}-\frac{x^{3}}{4} \leq-x+\frac{x^{2}}{2}$ for $x \in[0,0.42]$. So for $\gamma<\frac{1}{6}$,

$$
\boldsymbol{D}_{\mathrm{KL}}\left(\mathbb{P}_{\nu^{(1)}} \| \mathbb{P}_{\nu^{(j)}}\right) \leq 12 \gamma^{2} \mathbb{E}_{\nu^{(1)}}\left[T_{j}(\tau)\right] .
$$

Now define the events

$$
\begin{aligned}
A & =\{\tau<\infty\} \cap\{\hat{k} \neq j\} \\
A^{c} & =\{\tau=\infty\} \cup\{\hat{k}=j\} .
\end{aligned}
$$

Then since $A^{c} \subseteq\{\hat{k} \neq 1\}$ and $\pi$ is $\delta$-PAC policy we have $\mathbb{P}_{\nu^{(1)}}\left(A^{c}\right)+\mathbb{P}_{\nu^{(j)}}(A) \leq 2 \delta$. Now, by the Bretagnolle-Huber Inequality [1], Theorem 14.2],

$$
\begin{aligned}
2 \delta & \geq \frac{1}{2} \exp \left(-\boldsymbol{D}_{\mathrm{KL}}\left(\mathbb{P}_{\nu^{(1)}} \| \mathbb{P}_{\nu^{(j)}}\right)\right) \\
& \geq \frac{1}{2} \exp \left(-12 \gamma^{2} \mathbb{E}_{\nu^{(1)}}\left[T_{j}(\tau)\right]\right) .
\end{aligned}
$$

By rearranging and using 277 to get an upper bound on $\gamma$ in terms of the gap $\Delta_{i}$

$$
\begin{aligned}
\mathbb{E}_{\nu^{(1)}}\left[T_{j}(\tau)\right] & \geq \frac{1}{15 \gamma^{2}} \log \left(\frac{1}{4 \delta}\right) \\
& \geq \frac{3}{100 \Delta_{i}^{2}}(1-q)^{2} \log \left(\frac{1}{4 \delta}\right) .
\end{aligned}
$$

Repeating the argument for each $j \in\{2,3, \ldots K\}$ we get

$$
\begin{aligned}
\mathbb{E}_{\nu^{(1)}}[\tau] & =\sum_{j=1, j \neq i^{*}}^{K} \mathbb{E}\left[T_{j}(\tau)\right] \\
& \geq \sum_{j=1, j \neq i^{*}}^{K} \frac{3(1-q)^{2}}{100 \Delta_{j}^{2}} \log \left(\frac{1}{4 \delta}\right),
\end{aligned}
$$

and 36 gives the bound of the theorem.

Remark 1. We leave proving an instance-based lower bound as future work. We believe this will be quite challenging, since knowing only the value of the gap at quantile q gives only local information about the CDF of the distribution.

\section{A Private Algorithm FOR BEST-QUANTILE-ARM IDENTIFICATION}

We now turn to the privacy-preserving version of our bestarm identification algorithm. Bandit problems using private data arise naturally in medical and financial contexts, and privacy for online/sequential learning problems remains an 
active area of research. We provide results in this section on differentially private bandit learning. In differential privacy, the privacy guarantees should hold for any value of the input data. However, utility guarantees are made under the assumption that the rewards come from a stochastic process. For bandit learning, this means that our privacy guarantees will hold any realization of the arms' rewards and our bound on the number of pulls will depend on the distribution of the arms' rewards. The monograph of Dwork and Roth [70] provides an excellent introduction to the fundamentals of differential privacy.

To derive our privacy results, we think of the rewards from each of the arms at each time $t$ as coming from different individuals. This means that to protect an individual we are interested in event-level privacy, defined for private algorithms operating on streams [67]. Let $\mathbf{X}=\left[\mathbf{X}_{1}, \mathbf{X}_{2}, \ldots, \mathbf{X}_{K}\right]^{\top}$ be a collection of $K$ (infinite) sequences of rewards and let the $n$-th reward of arm $i$ be denoted by $\left(X_{n}^{i}\right)$.

Definition 3. Two sequences of rewards $\mathbf{X}=\left(X_{n}^{i}\right)_{i, n}$ and $\mathbf{X}^{\prime}=\left(X_{n}^{\prime i}\right)_{i, n}$ are called neighboring (denoted by $\mathbf{X} \sim \mathbf{X}^{\prime}$ ) if there exists only a single pair $(i, n)$ for which $X_{n}^{i} \neq X_{n}^{\prime i}$.

We note that this definition of neighboring for differentially private bandit problems is standard [68, 69] and we use the model of differential privacy under continual observation to handle the streaming setting.

Definition 4. A randomized algorithm $A$ is said to be $\epsilon$ differentially private $(\epsilon-\mathrm{DP})$ under continual observation if for any two neighboring rewards $\mathbf{X}$ and $\mathbf{X}^{\prime}$ and for any set $\mathcal{S}$ of outputs of the algorithm, we have $\operatorname{Pr}[A(\mathbf{X}) \in \mathcal{S}] \leq$ $e^{\epsilon} \operatorname{Pr}\left[A\left(\mathbf{X}^{\prime}\right) \in \mathcal{S}\right]$.

We can view $\mathbf{X}$ as a sequence of column vectors of rewards indexed by time. In the continual observation setting [67] the algorithm accesses these vectors sequentially (one entry per column based on the arm chosen by the algorithm) and hence the output $A(\mathbf{X})$ is also revealed one pull at a time. More specifically, a private bandit algorithm reveals which arm it chooses to pull at each time, so the overall output of an algorithm for best-arm identification is the sequence of pulled arm indices as well as the identified best arm. The advantage of the streaming definition [67] is that the algorithm's privacy guarantees can be made for pairs of neighboring streams $\mathbf{X}$ and $\mathbf{X}^{\prime}$ without the algorithm having to know termination time in advance. That is, when the algorithm terminates and the output is fully revealed, it guarantees the same probability bound to every pair of neighboring streams $\mathbf{X}$ and $\mathbf{X}^{\prime}$.

Differentially private algorithms are randomized in order to guarantee that the outputs do not depend too strongly on individual data points in the input. This randomization is internal to the algorithm: the privacy guarantee has to hold for any pair of neighboring input streams $\mathbf{X}$ and $\mathbf{X}^{\prime}$. The guarantee implies that an adversary, when viewing the output of the algorithm, will not be able to infer whether the input data was $\mathbf{X}$ or $\mathbf{X}^{\prime}$, even if all of the common entries of $\mathbf{X}$ and $\mathbf{X}^{\prime}$ are revealed. This is a strong guarantee which has led to a large body of work on differentially private learning.

Since differential privacy is a property of algorithms, a common approach to privately approximating a statistic (sometimes called a query) $f(\mathbf{X})$ is to compute $f(\mathbf{X})$ and add noise. A fundamental quantity of interest is the global sensitivity $G(f)=\max _{\mathbf{X} \sim \mathbf{X}^{\prime}}\left|f(\mathbf{X})-f\left(\mathbf{X}^{\prime}\right)\right|$, which measures how much $f(\mathbf{X})$ can change between neighboring inputs. If $G(f) \leq 1$ then the algorithm which outputs $f(\mathbf{X})+Z$ is $\epsilon$ differentially private if $Z$ has a Laplace distribution $\operatorname{Lap}(1 / \epsilon)$ with density $\frac{\epsilon}{2} e^{-\epsilon|z|}$. Unfortunately, the quantile functions (or quantile queries) have a very high global sensitivity. Taking the median query as an example, changing a single sample (in the worst case) can change the median of the set $\{0,0,0, M, M\}$ from 1 to $M$, meaning $G(f)=M$, which is full range of the data. As we discuss below, this makes privately computing quantiles challenging.

Differentially private algorithms also enjoy certain composition properties [70] (which we will use in the analysis of our proposed bandit scheme) that make them attractive for use in privacy settings. The first is basic composition: if $A_{1}$ and $A_{2}$ are $\epsilon_{1}-$ and $\epsilon_{2}$-DP algorithms resp., then for any $\mathbf{X}$ releasing the pair $\left\{A_{1}(\mathbf{X}), A_{2}(\mathbf{X})\right\}$ is $\left(\epsilon_{1}+\epsilon_{2}\right)$-DP (provided both algorithms use independent randomization). Parallel composition implies that if $A$ is an $\epsilon$-DP algorithm, then for any input $S$ and any column-wise partition of $\mathbf{X}$ into $\mathbf{X}_{1}, \mathbf{X}_{2}$, outputting $\left\{A\left(\mathbf{X}_{1}\right), A\left(\mathbf{X}_{2}\right)\right\}$ is $\epsilon$-DP (again, provided both algorithms are run using independent randomization).

\section{A. Differential privacy and quantiles}

Releasing a differentially private estimate of the $q$-quantile of a given distribution is considered to be a hard task. Tight bounds for $\epsilon$-differential privacy were given by Beimel, Nissim, and Stemmer [25] and Feldman and Xiao [26], with the accuracy dependent on the cardinality of the distribution's support. This makes the problem infeasible for continuous distributions such as those supported on $[0,1]$. The algorithm we propose gets around this by never publishing an approximation for $q$-quantile; instead we output an arm $\hat{k}$ that should have a higher $q$-quantile than any other arm $i$. To do this, we eliminate an arm by (privately) estimating the number of pairs of draws attesting for an arm's suboptimal $q$-quantile. This function/query is a counting query, whose global sensitivity is always 1 regardless of the size of the support of the reward distribution of arm $i$. This reformulation is what allows us to obtain a sample complexity bound that is independent of the support size of any arm's distribution and hence works for continuous distributions, even with unbounded support.

On the difficulties with a private UCB quantile algorithm. Differentially private UCB algorithms for the mean using treebased algorithms [66, 67] do not extend straightforwardly to the quantile case, but a carefully designed counting query ${ }^{6}$ makes using tree-based algorithms feasible to our problem. However, our proposed Algorithm 2 is superior to this approach in two respects, both related to the horizon $T$. First, (as observed by Sajed and Sheffet [69]) the tree based algorithm's utility bound has a poly $(\log (T))$ dependence whereas our algorithm's is only $\log \log (T) 7$ Secondly, the tree-based algorithms require

\footnotetext{
${ }^{6}$ Count the number of examples required to make the quantile-UCB of this arm the max.

${ }^{7}$ Both utility guarantees also have a $\log (1 / \delta)$-factor.
} 
knowing $T$ in advance; this is nontrivial because doubling tricks require either rebudgeting $\epsilon$ (incurring increased sample complexity) or discarding all samples when the next epoch begins, which incurs $\tilde{O}\left(\Delta_{i}^{-2}\right)$ pulls per suboptimal arm in every epoch because the UCB algorithm never eliminates any arms. Our gap definition and algorithm avoids having any such prior knowledge of $T$ or the value of the gap.

Notation. Throughout this section we deal with pure $\epsilon$-DP and use $\delta$ to represent the failure probability of our algorithm. The reader is advised to not be confused with the notion of $(\epsilon, \delta)-\mathrm{DP}^{8}$

\section{B. Differentially Private Successive Elimination for the Highest Quantile Arm}

The differentially private algorithm is shown in Algorithm 2. Much like the algorithm in Sajed and Sheffet [69], our algorithm is also epoch based. In epoch $e$ our goal is to eliminate all arms $i$ with gap (from (6) $\Delta_{i} \geq \Gamma_{e}=2^{-e}$ As we argue, the number of arm pulls in each epoch from each existing arm is $n_{e} \geq \Gamma_{e}^{-2}$. The key point is that due to the geometric nature of $\Gamma_{e}$ it follows that each $n_{e}$ is proportional to the sum of pulls thus far $\sum_{1<e^{\prime}<e} n_{e^{\prime}}$, and so we may as well split the stream into different chunks, starting each epoch anew (discarding all examples drawn in all previous epochs). Because we eliminate arms, this still doesn't cost us a lot in the number of overall pulls, yet allows us to avoid splitting the privacy budget $\epsilon$ due to parallel composition.

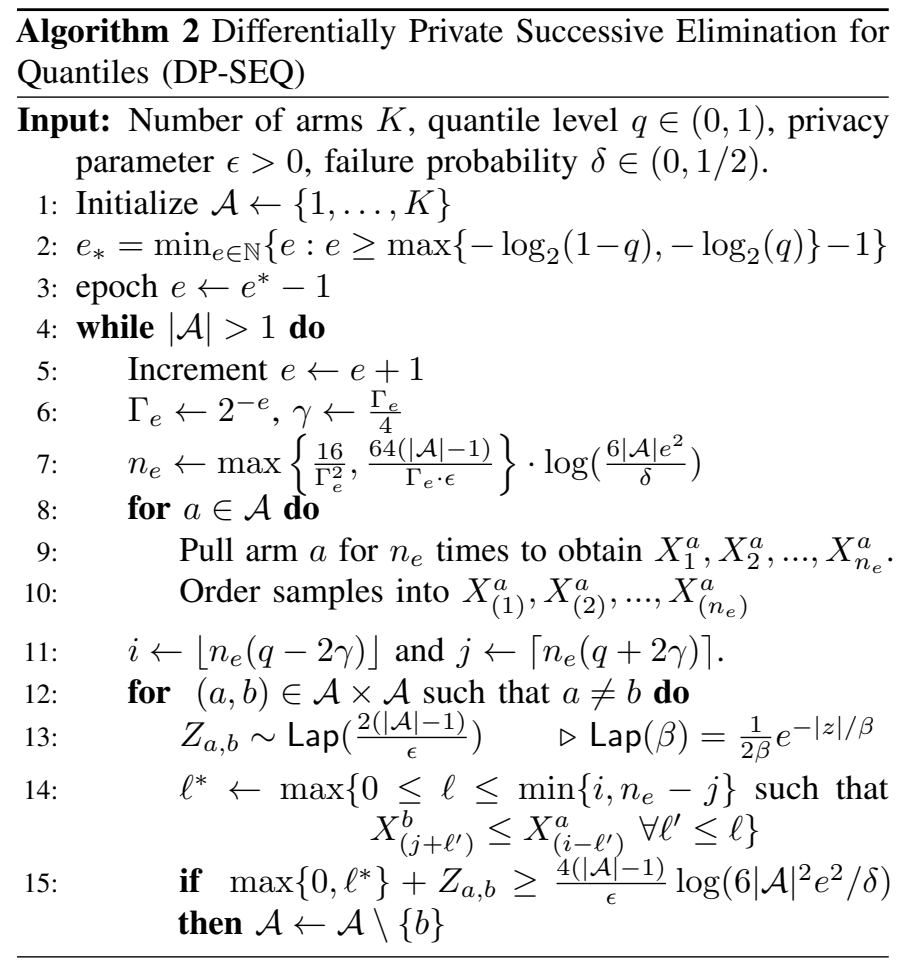

We still need a way to privately eliminate arms at the end of each epoch. In the case of the means, Sajed and Sheffet [69] eliminate arms by computing $\epsilon$-DP approximations of the means

\footnotetext{
${ }^{8} \mathrm{We}$ could have used the notion of approximate $(\epsilon, \delta)$-DP to reduce our total privacy loss by a factor of $\sqrt{K}$ by relaying on the advanced composition theorem $[71,72]$. As a matter of style, we opted for pure-DP.
}

and comparing those, leveraging the post-processing invariance of DP. Unfortunately, we cannot find $\epsilon$-DP approximations for $q$-quantiles that do not depend on the cardinality of the support. Instead, we resort to the more naive approach of pairwise comparisons between all $K(K-1) / 2=\Theta\left(K^{2}\right)$ pairs of arms. This requires partitioning the $\epsilon$ of our privacy budget into $\epsilon / 2(K-1)$ as each arm participates in at most $2(K-$ $1)$ many comparisons. However, using pairwise comparisons we are able to convert the higher-quantile question into a counting query: how many consecutive examples satisfy that $X_{(\mathrm{LCB}-i)}^{a} \geq X_{(\mathrm{UCB}+i)}^{b}$ ? Here LCB is the index of the lower confidence bound and UCB of the upper confidence bound. We prove that under event-level privacy, this query has sensitivity of at most 1 , allowing us to eliminate the suboptimal arm $b$ via the standard Laplace mechanism.

Our first result for differential privacy is a guarantee for Algorithm 2

Theorem 6. Algorithm 2 is $\epsilon$-differentially private under continual observation.

Proof of Theorem 6 Let $A$ denote the algorithm. Fix two neighboring input streams $\mathbf{X}$ and $\mathbf{X}^{\prime}$ and suppose that they differ in the entry $X_{n}^{a}$ corresponding to time $n$ and arm $a$. Let $e$ denote the epoch in which the time index $n$ falls. Since the rewards are identical up to epoch $e$, the distribution of outputs of $A(\mathbf{X})$ and $A\left(\mathbf{X}^{\prime}\right)$ are identical up to epoch $e$. In comparing the probabilities under inputs $\mathbf{X}$ and $\mathbf{X}^{\prime}$ we may therefore condition on the set $\mathcal{A}$ of available arms at the beginning of epoch.

Now let us consider epoch $e$ for the stream $\mathbf{X}$ and define $i$ and $j$ as in Algorithm 2. After each epoch we compare each pair of arms, so consider a pair of arms $a$ and $b$. If neither $X_{(j)}^{a} \leq X_{(j)}^{b}$ nor $X_{(j)}^{b} \leq X_{(i)}^{a}$, then set $\ell^{*}=0$. Otherwise without loss of generality assume $X_{j}^{b} \leq X_{i}^{a}$ and set $\ell^{*}$ to be the smallest element of the set $\max \left\{0 \leq \ell \leq \min \left\{i, n_{e}-\right.\right.$ $\left.j\}: \forall \ell^{\prime} \leq \ell X_{\left(j+\ell^{\prime}\right)}^{b} \leq X_{\left(i-\ell^{\prime}\right)}^{a}\right\}$. We claim this function has global sensitivity 1 .

We must evaluate how much $\ell^{*}$ can change by changing one sample from $\mathbf{X}$ to a neighboring $\mathbf{X}^{\prime}$ with index $\ell^{\prime *}$. Without loss of generality, assume the shifted reward is in arm $a$ so the rewards on arm $b$ are identical. We have the following sequence of inequalities for $\ell^{*}$

$$
\begin{aligned}
X_{(j)}^{b} \leq X_{(j+1)}^{b} \leq \ldots & \leq X_{\left(j+\ell^{*}\right)}^{b} \leq X_{\left(i-\ell^{*}\right)}^{a} \\
& \leq X_{\left(i-\ell^{*}+1\right)}^{a} \leq \ldots \leq X_{(i)}^{a} \leq X_{(i+1)}^{a} .
\end{aligned}
$$

Since $\ell^{*}$ is maximal, we know that $X_{\left(j+\ell^{*}+1\right)}^{b}>X_{\left(i-\ell^{*}-1\right)}^{a}$, giving us the chain of inequalities

$$
X_{\left(j+\ell^{*}+2\right)}^{b} \geq X_{\left(j+\ell^{*}+1\right)}^{b}>X_{\left(i-\ell^{*}-1\right)}^{a} \geq X_{\left(i-\ell^{*}-2\right)}^{a} .
$$

Now consider the rewards in $\mathbf{X}^{\prime}$ and the set of indices $\mathcal{T}=$ $\left[i-\ell^{*}-1, i\right]$. The sets $\left\{X_{(t)}^{\prime a}: t \in \mathcal{T}\right\}$ and $\left\{X_{(t)}^{a}: t \in \mathcal{T}\right\}$ differ in at most a single element. If they do not differ then they satisfy (37) and (38) so $\ell^{\prime *}=\ell^{*}$. If they do differ then the two sequences

$$
\begin{aligned}
& X_{\left(i-\ell^{*}-1\right)}^{a} \leq X_{\left(i-\ell^{*}\right)}^{a} \leq X_{\left(i-\ell^{*}+1\right)}^{a} \leq \ldots \leq X_{(i)}^{a} \leq X_{(i+1)}^{a} \\
& X_{\left(i-\ell^{*}-1\right)}^{\prime a} \leq X_{\left(i-\ell^{*}\right)}^{\prime a} \leq X_{\left(i-\ell^{*}+1\right)}^{\prime a} \leq \ldots \leq X_{(i)}^{\prime a} \leq X_{(i+1)}^{\prime a}
\end{aligned}
$$


are shifted by at most one position. Suppose that $X_{\left(j+\ell^{*}\right)}^{\prime b}>$ $X_{\left(i-\ell^{*}\right)}^{\prime a}$. Since $X_{\left(j+\ell^{*}\right)}^{\prime b}=X_{\left(j+\ell^{*}\right)}^{b} \leq X_{\left(i-\ell^{*}\right)}^{a}$, this implies $X_{\left(i-\ell^{*}\right)}^{a}>X_{\left(i-\ell^{*}\right)}^{\prime a}$. Since the sequences are shifted by at most 1 , we have $X_{\left(i-\ell^{*}\right)}^{a} \leq X_{\left(i-\ell^{*}+1\right)}^{\prime a}$. Then we have $X_{\left(j+\ell^{*}-1\right)}^{\prime b} \leq$ $X_{\left(i-\ell^{*}\right)}^{a} \leq X_{\left(i-\ell^{*}+1\right)}^{\prime a}$ which implies $\ell^{\prime *} \geq \ell^{*}-1$.

Now suppose $X_{\left(j+\ell^{*}+1\right)}^{\prime b} \leq X_{\left(i-\ell^{*}-1\right)}^{\prime a}$, which implies that $X_{\left(i-\ell^{*}-1\right)}^{a}<X_{\left(i-\ell^{*}-1\right)}^{\prime a}$. Since the sequences are shifted by at most $1, X_{\left(i-\ell^{*}-2\right)}^{\prime a}<X_{\left(i-\ell^{*}-1\right)}^{a}$ and we have $X_{\left(j+\ell^{*}+2\right)}^{\prime b}>$ $X_{\left(i-\ell^{*}-1\right)}^{a}>X_{\left(i-\ell^{*}-2\right)}^{\prime a}$, showing that $\ell^{\prime *} \leq \ell^{*}+1$.

We have shown that the function $\ell^{*}(\mathbf{X})$ has sensitivity 1 , so we can apply the Laplace noise mechanism. Define $\epsilon^{\prime} \triangleq$ $\epsilon / 2(|\mathcal{A}|-1)$. It follows then that the differentially private approximation $\ell^{*}+\operatorname{Lap}\left(1 / \epsilon^{\prime}\right)$ preserves $\epsilon^{\prime}$-DP. Since arm $a$ participates in at most $2(|\mathcal{A}|-1)$ many such queries in epoch $e$, we have by direct composition that our algorithm is $\epsilon$-DP.

We continue by providing a high probability guarantee on the first epoch for which the private SEQ (Algorithm 2) terminates.

Theorem 7. For Algorithm 2 the following events occur with probability at least $1-\delta:(a)$ it keeps at least one optimal arm in $\mathcal{A}$ and (b) it removes each suboptimal arm a by epoch $e=\left\lceil\log _{2}\left(1 / \Delta_{a}\right)\right\rceil$.

Proof of Theorem 7. Fix an epoch $e$, constant $\Gamma_{e}=2^{-e}$ and let $\delta>0$. We denote the following "bad" events at the end of the epoch,

$$
\begin{aligned}
& E_{1} \triangleq\left\{\exists a \in \mathcal{A}: X_{(i)}^{a}>F_{a}^{-1}\left(i / n_{e}+\Gamma_{e} / 4\right)\right. \\
& \left.\quad \text { or } X_{\left(\left\lfloor i-n_{e} \frac{\Gamma_{e}}{4}\right\rfloor\right)}^{a}<F_{a}^{-1}\left(i / n_{e}-\Gamma_{e} / 4\right)\right\}, \\
& E_{2} \triangleq\left\{\exists a \in \mathcal{A}: X_{(j)}^{a}<F_{a}^{-1}\left(j / n_{e}-\Gamma_{e} / 4\right)\right. \\
& \left.\quad \text { or } X_{\left(\left\lceil j+n_{e} \frac{\Gamma_{e}}{4}\right\rceil\right)}^{a}>F_{a}^{-1}\left(j / n_{e}+\Gamma_{e} / 4\right)\right\}, \\
& E_{3} \triangleq\left\{\exists(a, b) \in \mathcal{A}^{2}:\left|Z_{a, b}\right|>\frac{2(|\mathcal{A}|-1)}{\epsilon} \log \left(6|\mathcal{A}|^{2} e^{2} / \delta\right)\right\} .
\end{aligned}
$$

We have $n_{e} \geq \frac{16}{\Gamma_{e}^{2}} \log \left(\frac{6|\mathcal{A}| e^{2}}{\delta}\right) \geq \frac{8}{\Gamma_{e}^{2}} \log \left(\frac{12|\mathcal{A}| e^{2}}{\delta}\right)$, so we can apply Lemma 1 (Appendix B with $\zeta=\Gamma_{e} / 4$ to show that for a given arm $a$ and any specific index $k$, it holds that

$$
\begin{aligned}
& \operatorname{Pr}\left[X_{(k)}^{a}<F_{a}^{-1}\left(k / n_{e}-\frac{\Gamma_{e}}{4}\right)\right] \leq \frac{\delta}{12 e^{2}|\mathcal{A}|}, \\
& \operatorname{Pr}\left[X_{(k)}^{a}>F_{a}^{-1}\left(k / n_{e}+\frac{\Gamma_{e}}{4}\right)\right] \leq \frac{\delta}{12 e^{2}|\mathcal{A}|} .
\end{aligned}
$$

Applying the union bound over the $|\mathcal{A}|$ choices for an arm and the two particular indices $k=i$ and $k=\left\lfloor i-n_{e} \frac{\Gamma_{e}}{4}\right\rfloor$, we have that $\operatorname{Pr}\left[E_{1}\right] \leq \delta / 6 e^{2}$. Similarly, the same line of reasoning gives that $\operatorname{Pr}\left[E_{2}\right] \leq \delta / 6 e^{2}$. Lastly, due to the properties of the Laplace distribution (or the exponential distribution which dictates the magnitude of $\left|Z_{a, b}\right|$ we have that $\operatorname{Pr}\left[E_{3}\right] \leq|\mathcal{A}|^{2} \delta / 6 e^{2}|\mathcal{A}|^{2}=\delta / 6 e^{2}$. We apply the union bound again (twice) to infer that $\operatorname{Pr}\left[E_{1} \cup E_{2} \cup E_{3}\right] \leq \delta / 2 e^{2}$, and thus, the probability that

$$
\operatorname{Pr}\left[\exists e: E_{1}, E_{2} \text { or } E_{3} \text { occur }\right] \leq \sum_{e \geq 0} \delta / 2 e^{2} \leq \delta .
$$

We continue under the assumption that in all epochs all three bad events never occur. Also by our choice of $n_{e}$ it is true that $8(|\mathcal{A}|-1) \epsilon^{-1} \log \left(6|\mathcal{A}|^{2} e^{2} / \delta\right) \leq 16(|\mathcal{A}|-$ 1) $\epsilon^{-1} \log \left(6|\mathcal{A}| e^{2} / \delta\right) \leq n_{e} \Gamma_{e} / 4$. It is now fairly straightforward to argue that when comparing a suboptimal arm $a$ and an optimal arm $b$ we never remove $b$ : this follows from the fact that in this case we have

$$
\begin{aligned}
X_{(j)}^{b} & \geq F_{b}^{-1}\left(\frac{j}{n_{e}}-\frac{\Gamma_{e}}{4}\right) \\
& \geq F_{b}^{-1}(q)>F_{a}^{-1}(q) \geq F_{a}^{-1}\left(\frac{i}{n_{e}}+\frac{\Gamma_{e}}{4}\right) \geq X_{(i)}^{a}
\end{aligned}
$$

and so for such a pair $\ell^{*}=0$, making $\ell^{*}+Z_{a, b} \leq$ $2(|\mathcal{A}|-1) \log \left(6|\mathcal{A}|^{2} e^{2} / \delta\right) / \epsilon$ under the complement of $E_{3}$. Thus, we can only eliminate an optimal arm when comparing it to another optimal arm, and so $\mathcal{A}$ must always contain at least one optimal arm. Secondly, when comparing an optimal arm $a$ to a suboptimal arm $b$ where the optimality gap is at least $2^{-e}$ we have that at epoch $e$ it holds that for $\ell=6(|\mathcal{A}|-1) \log \left(6|\mathcal{A}|^{2} e^{2} / \delta\right) \epsilon$ we have

$$
\begin{aligned}
X_{(j)}^{b} \leq X_{(j+1)}^{b} \leq \ldots & \leq X_{(j+\ell)}^{b} \\
& \leq X_{\left(\left\lceil j+n_{e} \frac{\Gamma_{e}}{4}\right)\right\rceil}^{b} \leq F_{b}^{-1}\left(q+\Gamma_{e}\right)
\end{aligned}
$$

and

$$
\begin{aligned}
F_{b}^{-1}\left(q+\Gamma_{e}\right) \leq F_{a}^{-1}\left(q-\Gamma_{e}\right) & \leq X_{\left(\left\lfloor i-n_{e} \frac{\Delta}{4}\right\rfloor\right)}^{a} \\
& \leq X_{(i-\ell)}^{a} \leq \ldots \leq X_{(i)}^{a} .
\end{aligned}
$$

It follows that for such a pair $\ell^{*} \geq 6(|\mathcal{A}|-1) \log \left(6|\mathcal{A}|^{2} e^{2} / \delta\right) / \epsilon$, under $E_{3}$ we have that $\ell^{*}+Z_{a, b} \geq 6(|\mathcal{A}|-1) \log \left(4|\mathcal{A}|^{2} e^{2} / \delta\right) / \epsilon$ so we eliminate $\operatorname{arm} b$. The latter, (42) and (43) complete the proof.

Lastly, we characterize the sample complexity of DP-SEQ (Algorithm 2), the number of pulls for each suboptimal arm and the total number of pulls at termination.

Theorem 8. With probability at least $1-\delta$, Algorithm 2, pulls each suboptimal arm a at most

$$
\mathcal{O}\left(\left(\frac{1}{\Delta_{a}^{2}}+\frac{K}{\epsilon \Delta_{a}}\right) \log \left(\frac{K}{\delta} \log \left(\frac{1}{\Delta_{a}}\right)\right)\right)
$$

many times.

By taking $\epsilon \rightarrow \infty$, Theorem 8 provides the utility of the standard (non-private) epoch-based successive elimination variant of Algorithm 1. Indeed, by introducing epochs the concentration bound in (5) becomes

$$
\mathbb{P}\left(F_{i}^{-1}(q) \in\left[X_{\left(\left\lfloor n_{e}\left(q-\mathrm{D}_{e}\right)\right\rfloor\right)}^{i}, X_{\left(\left\lceil n_{e}\left(q+\mathrm{D}_{e}\right)\right\rceil\right)}^{i}\right]\right)>1-\frac{\delta}{2 K e^{2}},
$$

$e$ denotes the epoch, $\mathrm{D}_{e}=2^{-e}$ and $n_{e}=\mathrm{D}_{e}^{-2} \log \left(4 K e^{2} / \delta\right) / 2$. This yields a bound on the total number of pulls for the epochbased algorithm of the order of

$$
\mathcal{O}\left(\sum_{i \in \mathcal{A}^{-i^{*}}} \frac{1}{\Delta_{i}^{2}} \log \left(\frac{K}{\delta} \log \left(\frac{1}{\Delta_{i}}\right)\right)\right),
$$

matching the ( $\varepsilon$-optimal) bounds of [24]. As a consequence of the epoch-based approach, the dependence $\log \left(K /\left(\delta \Delta_{i}\right)\right)$ in (13) becomes $\log \left(K \delta^{-1} \log \left(\Delta_{i}^{-1}\right)\right)$ for $i \in \mathcal{A} \backslash\left\{i^{*}\right\}$. However, 
this comes at the expense of much larger constants. We continue by presenting the proof of Theorem 8

Proof of Theorem 8 Fix any suboptimal arm $a$. Denote $e^{*}$ as the first integer $e$ for which $2^{-e} \leq \Delta_{a}$. Thus $\Delta_{e^{*}}=2^{-e^{*}} \leq$ $\Delta_{a}<2 \Delta_{e^{*}}$ making $2^{e^{*}} \leq 2 / \Delta_{a}$. According to Theorem 7 we have that with probability at least $1-\delta$ by epoch $e^{*}$ arm $a$ is eliminated. Since in any epoch we have that $|\mathcal{A}| \leq K$, we have that the total number of pulls of arm $a$ is

$$
\begin{aligned}
& \sum_{0 \leq e \leq e^{*}} n_{e} \\
\leq & \sum_{0 \leq e \leq e^{*}}\left(\frac{16}{\Gamma_{e}^{2}}+\frac{64(K-1)}{\Gamma_{e} \cdot \epsilon}\right) \cdot \log \left(\frac{6 K e^{2}}{\delta}\right) \\
\leq & 16 \log \left(\frac{6 K\left(e^{*}\right)^{2}}{\delta}\right) \sum_{0 \leq e \leq e^{*}} 2^{2 e}+\frac{64 K \log \left(\frac{6 K\left(e^{*}\right)^{2}}{\delta}\right)}{\epsilon} \sum_{0 \leq e \leq e^{*}} 2^{e} \\
\leq & 32 \log \left(\frac{6 K\left(e^{*}\right)^{2}}{\delta}\right) 2^{2 e^{*}}+\frac{128 K \log \left(\frac{6 K\left(e^{*}\right)^{2}}{\delta}\right)}{\epsilon} 2^{e^{*}} \\
\leq & \frac{128 \log \left(\frac{6 K\left(e^{*}\right)^{2}}{\delta}\right)}{\Delta_{a}^{2}}+\frac{256 K \log \left(\frac{6 K\left(e^{*}\right)^{2}}{\delta}\right)}{\epsilon \Delta_{a}} \\
\leq & \left(\frac{1}{\Delta_{a}^{2}}+\frac{K}{\epsilon \Delta_{a}}\right) \cdot 512 \log \left(\frac{6 K}{\delta} \cdot \log \left(\frac{1}{\Delta_{a}}\right)\right) .
\end{aligned}
$$

To conclude, the total number of samples (and pulls) is

$$
\mathcal{O}\left(\sum_{a \in \mathcal{A}^{-i^{*}}}\left(\frac{1}{\Delta_{a}^{2}}+\frac{K}{\epsilon \Delta_{a}}\right) \cdot \log \left(\frac{K}{\delta} \log \left(\frac{1}{\Delta_{a}}\right)\right)\right)
$$

with probability at least $1-\delta$.

\section{NUMERICAL ILLUSTRATIONS AND FURTHER DISCUSSION OF THE RESULTS}

In this section, we provide indicative numerical simulations (along with relevant discussion) exploring and confirming various properties related to the proposed elimination algorithms (private and non-private), as well as the proposed definition of the associated suboptimality gap.

a) Empirical verification of the tightness of the bounds:

To empirically validate our theoretical results on the sample complexity, we show in Figure 3 the average number of samples to identify the best arm for a Gaussian (left) and discrete (right) problem setting. For both settings the average number of pulls is evaluated through 100 independent runs. These curves show that there exists a constant $C$ such that the sample complexity of the algorithm matches our analysis, specifically $C=3 / 2$ for the left and right figure. For the Gaussian distribution, $\mu_{i^{*}}=\mu_{i}=0, \sigma_{i}=2$ while $\sigma_{i^{*}}$ varies. The discrete distribution is provided in Figure 2 on the left, $q=0.4$ while the levels $\ell_{1}, \ell_{3}$ vary (see Figure 2, left).

b) Sample complexity for heavy-tailed distributions is not expensive at all: In the following example, we consider two cases (Gaussian and log-normal distributions), for which the differences between the quantile values are different but the gap is identical for any $q \in(0,1)$. The latter can be verified by our Definition 6 As a consequence we expect to find the same
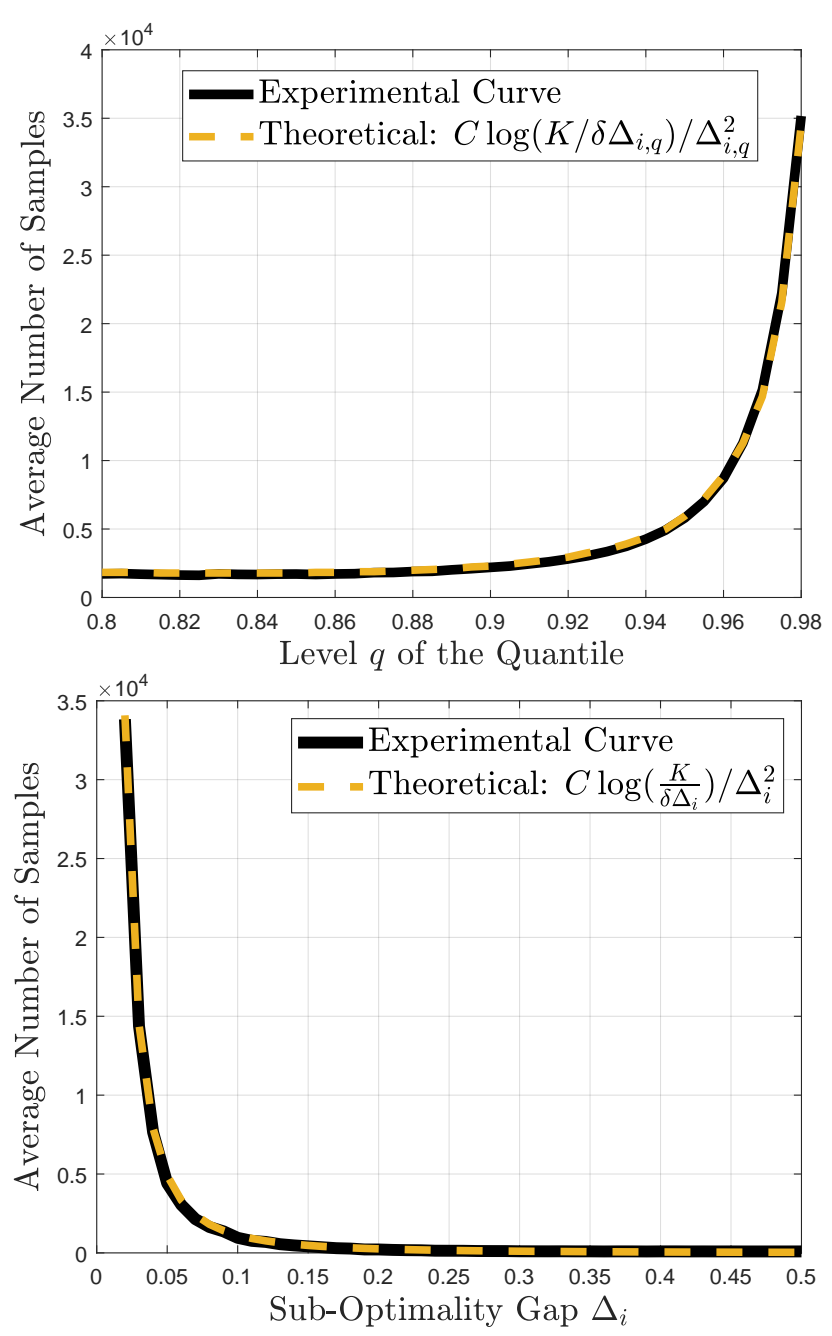

Fig. 3. Upper: Gaussian data, $\mu_{i^{*}}=\mu_{i}=0, \sigma_{i^{*}}=1$ and $\sigma_{i}=0.5$, illustration of the average number of samples (and pulls) as the level of $q$ increases. Lower: Discrete data, $q=0.4$, illustration of the average number of samples (and pulls) as the suboptimality gap $\Delta_{i}$ increases. In both cases we use 100 in total independent runs.

average number of pulls for Gaussian and log-normal quantile bandits in our experiment. We can see this by comparing the performance (average termination time averaged over 500 runs) for a normal distribution and a log-normal distribution for large values of $q$, see Figure 4 (left). We take $K=2$. The suboptimal distribution (normal or log-normal) has mean 0 and parameter $\sigma=2$. We vary the best arm by changing $\sigma$. In our definition of gap, the gap between two normal distributions with parameters $\sigma_{i}$ and $\sigma_{i^{*}}$ is the same as the gap between two log-normal distributions with parameters $\sigma_{i}$ and $\sigma_{i^{*}}$. Each curve shows that the sample complexity when comparing normal and lognormal distributions is the same. In the case of the log-normal distributions the difference in the $q$-quantiles may be quite large. However, the sample complexity of the algorithm depends on the gap.

c) The cost of privacy for Algorithm 17. Figure 4 (right) shows the performance of Algorithm 1 as a function of the privacy risk $\epsilon$. As expected, as $\epsilon$ increases the sample complexity decreases. The plots show that as the quantile decreases the gap in expected pulls between the private and non-private algorthms decreases. The high cost of privacy in 

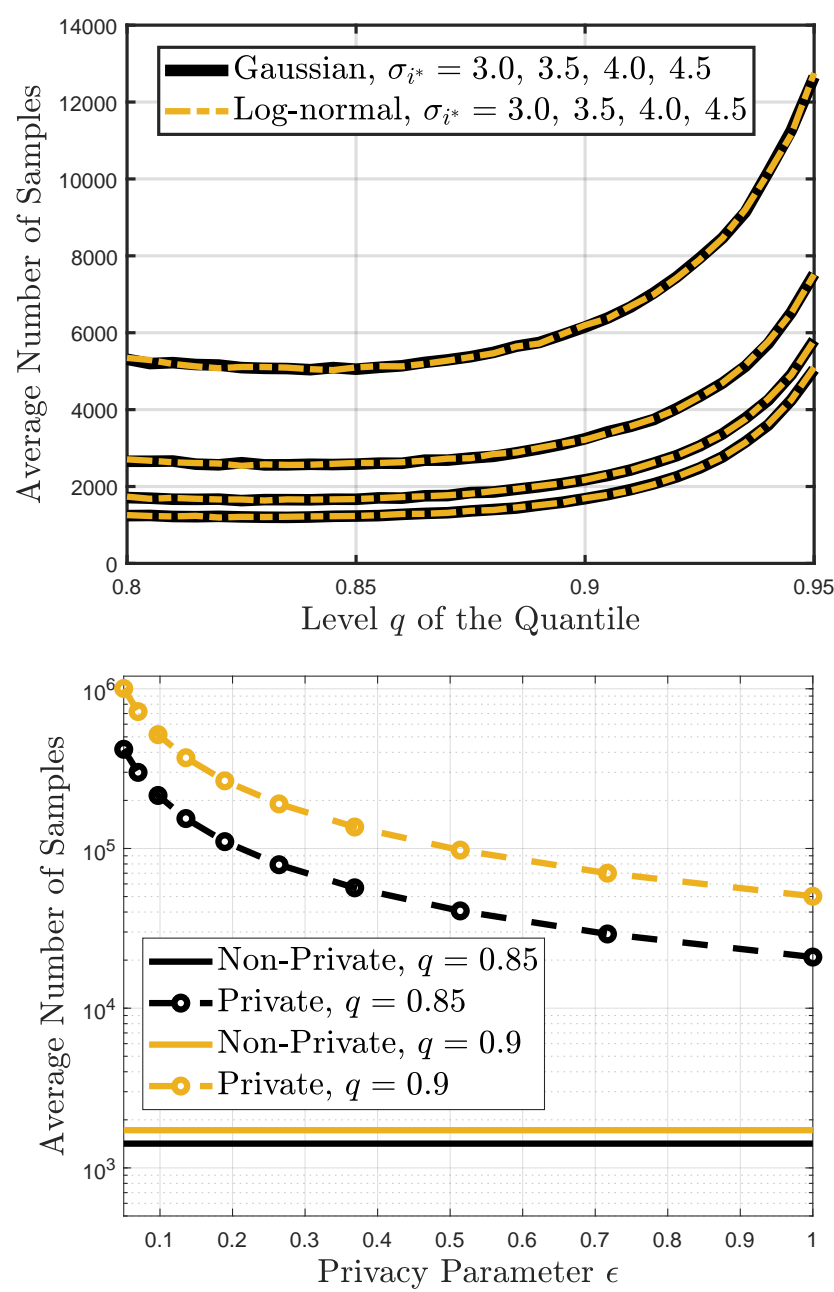

Fig. 4. Upper: Sample complexity of Algorithm 1 for Gaussian and $\log$ normal data, $K=2$. For both cases $\mu=0$ for all the arms, $\sigma_{i}=2$. Lower Comparison of the private (Algorithm 2) and non-private SEQ (Algorithm 11. We consider log-normal distributions with $K=10$ arms and parameters $\mu_{i^{*}}=\mu_{i}=0, \sigma_{i} \in[0.1,0.2, \ldots, 0.9]$ and $\sigma_{i^{*}}=2$. We compare the estimated number of samples (averaging over 10 iterations) for different values of the privacy parameter $\epsilon \in[0.05,1]$ and for quantile levels $q=0.85$ and $q=0.9$.

this example shows that there is potential for improvement in the private algorithm: in order to get the sample complexity scaling we chose to double epoch sizes (a standard technique) but empirically we may choose a less aggressive approach.

\section{Discussion AND Future Directions}

In this paper we characterized the sample complexity of the quantile multi-armed bandit problem when the goal is to exactly identify the arm with the highest $q$-quantile in terms of a new measure of suboptimality (gap) between the distributions of each pair of arms. The problem of the lowest $q$-quantile is a simple modification of our method. Motivated by scenarios where the arm rewards are private or carry sensitive information, we also provided the first differentially private algorithm for the quantile bandit problem. These privacy considerations lead to an interesting open problem which we discuss next.

Open Problem for Privacy. Algorithm 2 pulls each suboptimal arm $i$ roughly $K / \epsilon \Delta_{i}$ times more than Algorithm 1 Because we cannot publish approximations of the $q$-quantiles, the factor of $K$ comes because of the need to make private pairwise comparisons. An open question remains: can we avoid this factor of $K$ or is there a converse showing it is necessary? This factor does not appear when looking at the difference between private and non-private best mean arm identification. We would like to know if a different elimination procedure would have the same property but for the quantiles.

The bandit literature is vast, with many variations, and for some of these the quantile bandit setting might provide an interesting twist as a form of risk-aware learning. Bandit optimization with risk control is a particularly interesting direction to which this work can apply. For the case of contaminated quantiles [58] our results imply that $\leq \Delta_{i} / 2$ fraction of contaminated examples could be handled for general $q$-quantiles. There are still open fundamental questions one may ask, in particular related to the hardness of best-armidentification for functionals of the distribution beyond the mean and variance [53] in the private and non-private case.

\section{APPENDIX A \\ PROOF OF THEOREM 1}

We start by providing a lemma and then we continue with the proof of Theorem 1 .

Lemma 2. Let $F$ and $F^{\prime}$ be two distributions such that $d_{\mathrm{TV}}\left(F, F^{\prime}\right)=\eta$. Then for any $q \in(\eta, 1-\eta)$ it holds that $F^{-1}(q-\eta) \leq\left(F^{\prime}\right)^{-1}(q) \leq F^{-1}(q+\eta)$.

Proof. By definition, for any $x \in \mathbb{R}$ it holds that $\mid F(x)-$ $F^{\prime}(x) \mid \leq \eta$. Define the set $S(F, q)=\{\xi: F(\xi) \geq q\}$ where $F^{-1}(q)=\inf S(F, q)$. It follows that any $\xi \in S\left(F^{\prime}, q\right)$ also satisfies that $F(\xi) \geq F^{\prime}(\xi)-\eta \geq q-\eta$ which means $\xi \in S(F, q-\eta)$, and so $F^{-1}(q-\eta)=\inf S(F, q-\eta) \leq$ $\left(F^{\prime}\right)^{-1}(q)=\inf S\left(F^{\prime}, q\right)$. Similarly, any $\xi \in S(F, q+\eta)$ also belongs to the set $S\left(F^{\prime}, q\right)$ proving that $\left(F^{\prime}\right)^{-1}(q) \leq$ $F^{-1}(q+\eta)$.

\section{A. Proof of Theorem 1.}

First, recall the definition of the distance to flip $d_{\text {flip }}\left(F_{l}, F_{\mathrm{h}}\right)$ that is equal to

$$
\inf _{\left(G_{\mathrm{h}}, G_{1}\right):\left(G_{\mathrm{h}}\right)^{-1}(q)>G_{1}^{-1}(q)} \max \left\{d_{\mathrm{TV}}\left(F_{\mathrm{l}}, G_{\mathrm{h}}\right), d_{\mathrm{TV}}\left(F_{\mathrm{h}}, G_{1}\right)\right\}
$$

and the definition of the gap

$$
\Delta\left(F_{1}, F_{\mathrm{h}}\right)=\sup \left\{\eta \geq 0: F_{1}^{-1}(q+\eta) \leq F_{\mathrm{h}}^{-1}(q-\eta)\right\} .
$$

Now, given $\eta<\min \{q, 1-q\} / 2$ and a distribution $F$ we define two specific shifts. The first is referred to as $\eta$ push of $F$ and denoted $F^{\rightarrow \eta}$ - we subtract $\eta$ probability mass from the interval $\left(-\infty, F^{-1}(q)\right)$ and add $\eta$ probability mass to any point or interval in $\left(F^{-1}(q+2 \eta), \infty\right)$. It is now clear that the $q$-quantile of $F^{\rightarrow \eta}$ is in fact $F^{-1}(q+\eta)$ and that $d_{\mathrm{TV}}\left(F, F^{\rightarrow \eta}\right)=\eta$. The second shift is equivalent and is an $\eta$-pull, denoted $F^{\leftarrow \eta}-$ we subtract $\eta$-probability mass from the interval $\left(F^{-1}(q), \infty\right)$ and move it to the interval $\left(-\infty, F^{-1}(q-2 \eta)\right)$. One can check that the $q$-quantile of $F^{\leftarrow \eta}$ is in fact $F^{-1}(q-\eta)$ and that $d_{\mathrm{TV}}\left(F, F^{\leftarrow \eta}\right)=\eta$. 
We now prove the first part of the lemma. Denote that $\Delta\left(F_{1}, F_{\mathrm{h}}\right)=\Delta$. Namely, for every $\eta>0$ it holds that $F_{1}^{-1}(q+\Delta+\eta)>F_{\mathrm{h}}^{-1}(q-\Delta-\eta)$. For any $\eta>0$, consider the $(\Delta+\eta)$-push of $F_{1}$ so that $\left(F_{1}^{\rightarrow(\Delta+\eta)}\right)^{-1}(q)=F_{1}^{-1}(q+\Delta+\eta)$ and the $(\Delta+\eta)$-pull of $F_{\mathrm{h}}$ so that $\left(F_{\mathrm{h}}^{\leftarrow(\Delta+\eta)}\right)^{-1}(q)=$ $F_{\mathrm{h}}^{-1}(q-\Delta-\eta)$. Putting these inequalities together shows that $\left(F_{1}^{\rightarrow(\Delta+\eta)}\right)^{-1}(q)>\left(F_{\mathrm{h}}^{\leftarrow(\Delta+\eta)}\right)^{-1}(q)$. Applying the definition of the distance to quantile flip, this shows that $d_{\text {flip }}\left(F_{1}, F_{\mathrm{h}}\right)<$ $\Delta+\eta$ for any positive $\eta$. Thus, $d_{\text {flip }}\left(F_{1}, F_{\mathrm{h}}\right) \leq \Delta$. Specifically, in the case where $\Delta\left(F_{1}, F_{\mathrm{h}}\right)=\Delta=0$ we have that $d_{\text {flip }}\left(F_{1}, F_{\mathrm{h}}\right)=0$.

We now show the contrapositive. Assume that $\Delta\left(F_{1}, F_{\mathrm{h}}\right)>$ 0 . Fix any $0<\eta<\Delta\left(F_{1}, F_{\mathrm{h}}\right)$, and note that it holds that $F_{1}^{-1}(q+\eta) \leq F_{\mathrm{h}}^{-1}(q-\eta)$. Fix any $\tilde{G}$ and $\tilde{H}$ such that $d_{\mathrm{TV}}\left(F_{1}, \tilde{G}\right) \leq \eta$ and $d_{\mathrm{TV}}\left(F_{\mathrm{h}}, \tilde{H}\right) \leq \eta$. It follows from Lemma 2 and the definition of the gap that

$$
\tilde{G}^{-1}(q) \leq F_{1}^{-1}(q+\eta) \leq F_{\mathrm{h}}^{-1}(q-\eta) \leq H^{-1}(q) .
$$

This shows that any pair of distributions with max TV-distance of $\eta$ to $F_{1}$ and $F_{\mathrm{h}}$ is such that that the q-quantile has not flipped and it still holds that $\tilde{G}^{-1}(q) \leq \tilde{H}^{-1}(q)$, and so $d_{\text {flip }}\left(F_{1}, F_{\mathrm{h}}\right) \geq \eta$. Since we've shown that for all $\eta$ that satisfy $\eta<\Delta\left(F_{1}, F_{\mathrm{h}}\right)$ the inequality $d_{\text {flip }}\left(F_{\mathrm{l}}, F_{\mathrm{h}}\right) \geq \eta$ holds, it follows that $\left.d_{\text {flip }}\left(F_{1}, F_{\mathrm{h}}\right)\right) \geq \sup _{\eta}\left\{0<\eta<\Delta\left(F_{1}, F_{\mathrm{h}}\right)\right\}=\Delta\left(F_{1}, F_{\mathrm{h}}\right)$. The last completes the proof.

\section{APPENDIX B}

\section{Proof of Properties AND CONCENTRATION Bound}

We start by providing two inequalities for the quantile that we will use later.

Proposition 2. Fix $q \in(0,1)$. Let $F_{X}^{-1}(q)$ be the q-quantile of a random variable $X$ provided in Definition 11 Then

$$
\mathbb{P}\left[X<F_{X}^{-1}(q)\right] \leq q
$$

and

$$
\mathbb{P}\left[X>F_{X}^{-1}(q)\right] \leq 1-q .
$$

Proof. Let $x_{n}$ be a monotonically increasing sequence such that $\lim _{n \rightarrow \infty} x_{n}=F_{X}^{-1}(q)$, then

$$
\operatorname{Pr}\left[X<F_{X}^{-1}(q)\right]=\lim _{n \rightarrow \infty} \operatorname{Pr}\left[X \leq x_{n}\right]=\lim _{n \rightarrow \infty} F\left(x_{n}\right) .
$$

Consider $x_{n}=F_{X}^{-1}(q)-2^{-n}$ and assume for sake of contradiction that $\operatorname{Pr}\left[X<F_{X}^{-1}(q)\right]=q+\epsilon>q$ for some $\epsilon>0$. It follows that for some $n$ it holds that $F\left(x_{n}\right)>q+\epsilon / 2$. Assuming $\xi=x_{n}<F_{X}^{-1}(q)$ in the set $\{\xi: \operatorname{Pr}[X \leq \xi] \geq q\}$ contradicts the definition of $F_{X}^{-1}(q)$.

It is true that $\operatorname{Pr}\left[X>F_{X}^{-1}(q)\right]=1-\operatorname{Pr}\left[X \leq F_{X}^{-1}(q)\right]$. The second part of the claim follows from $\operatorname{Pr}\left[X \leq F_{X}^{-1}(q)\right] \geq q$, the definition of the quantile $F_{X}^{-1}(q)$ and the fact that the CDF $F_{X}(\cdot)$ is right continuous.

Theorem 9 (Concentration Bound). Choose a level $q \in(0,1)$. Fix $\delta \in(0,1)$. For any $n \in \mathbb{N}$, if

$$
\sqrt{\frac{\log (2 / \delta)}{2 n}} \leq \zeta \leq \min \{q, 1-q\}
$$

then

$$
\mathbb{P}\left(F_{X}^{-1}(q) \notin\left[X_{(\lfloor n(q-\zeta)\rfloor)}, X_{(\lceil n(q+\zeta)])]}\right]\right) \leq \delta .
$$

Proof. Hoeffding's inequality gives

$$
\begin{aligned}
& \mathbb{P}\left[\frac{1}{n} \sum_{i=1}^{n} \mathbf{1}_{X_{i}<F_{X}^{-1}(q)}<\mathbb{P}\left[X<F_{X}^{-1}(q)\right]+\sqrt{\frac{\log (2 / \delta)}{2 n}}\right] \\
& >1-\delta / 2
\end{aligned}
$$

and we have

$$
\begin{aligned}
& 1-\frac{\delta}{2} \\
& <\mathbb{P}\left[\frac{1}{n} \sum_{i=1}^{n} \mathbf{1}_{X_{i}<F_{X}^{-1}(q)}<\mathbb{P}\left[X<F_{X}^{-1}(q)\right]+\sqrt{\frac{\log (2 / \delta)}{2 n}}\right] \\
& \leq \mathbb{P}\left[\frac{1}{n} \sum_{i=1}^{n} \mathbf{1}_{X_{i}<F_{X}^{-1}(q)}<q+\sqrt{\frac{\log (2 / \delta)}{2 n}}\right] \\
& =\mathbb{P}\left[\sum_{i=1}^{n} \mathbf{1}_{X_{i}<F_{X}^{-1}(q)}<n\left(q+\sqrt{\frac{\log (2 / \delta)}{2 n}}\right)\right] \\
& \leq \mathbb{P}\left[\sum_{i=1}^{n} \mathbf{1}_{X_{i}<F_{X}^{-1}(q)}<\left\lceil n\left(q+\sqrt{\frac{\log (2 / \delta)}{2 n}}\right)\right]\right] \\
& \leq \mathbb{P}\left[\sum_{i=1}^{n} \mathbf{1}_{X_{i}<F_{X}^{-1}(q)}<\lceil n(q+\zeta)], \forall \zeta \geq \sqrt{\frac{\log (2 / \delta)}{2 n}}\right.
\end{aligned}
$$

and (52) comes from Proposition 2. The last inequality implies that for all $\zeta \geq \sqrt{\log (2 / \delta) / 2 n}$ the following holds

$$
\mathbb{P}\left[\sum_{i=1}^{n} \mathbf{1}_{X_{i}<F_{X}^{-1}(q)} \geq\lceil n(q+\zeta)\rceil\right] \leq \delta / 2 .
$$

It is true that

$$
\begin{aligned}
& X_{(\lceil n(q+\zeta)\rceil)}<F_{X}^{-1}(q) \\
& \Longleftrightarrow \sum_{i=1}^{n} \mathbf{1}_{X_{i}<F_{X}^{-1}(q)} \geq\lceil n(q+\zeta)\rceil,
\end{aligned}
$$

and 53 gives

$$
\mathbb{P}\left[X_{(\lceil n(q+\zeta)\rceil)}<F_{X}^{-1}(q)\right] \leq \delta / 2, \forall \zeta \geq \sqrt{\frac{\log (2 / \delta)}{2 n}} .
$$

Similarly, for all $\zeta \in[\sqrt{\log (2 / \delta) / 2 n}, q]$

$$
\begin{aligned}
& 1-\frac{\delta}{2} \\
& \leq \mathbb{P}\left[\sum_{i=1}^{n} \mathbf{1}_{X_{i}>F_{X}^{-1}(q)}<n-\lfloor n(q-\zeta)\rfloor\right] \\
& \leq \mathbb{P}\left[\sum_{i=1}^{n} \mathbf{1}_{X_{i}>F_{X}^{-1}(q)}<n-\max \{\lfloor n(q-\zeta)\rfloor, 1\}+1\right],
\end{aligned}
$$

and due to the definition of order statistics with restricted indices in the set $\{1,2, \ldots, n\}$ it is true that

$$
\begin{aligned}
& X_{(\lfloor n(q-\zeta)\rfloor)}>F_{X}^{-1}(q) \\
& \Longleftrightarrow \sum_{i=1}^{n} \mathbf{1}_{X_{i}>F_{X}^{-1}(q)} \geq n-\max \{\lfloor n(q-\zeta)\rfloor, 1\}+1 .
\end{aligned}
$$


Then (56) gives

$$
\mathbb{P}\left[X_{(\lfloor n(q-\zeta)\rfloor)}>F_{X}^{-1}(q)\right] \leq \delta / 2,
$$

for all $\zeta \in[\sqrt{\log (2 / \delta) / 2 n}, q]$. Finally, 55, (58) and the union bound give the statement of the lemma.

\section{APPENDIX C}

AnAlysis of the CASES $\Delta_{i}>0$ AND $\Delta_{i}=0$

The next corollary provides an analysis for the cases of strictly positive or zero gap. Note that there exists $A>0$ such that $F_{i^{*}}^{-1}(q-A) \geq F_{i}^{-1}(q+A)$ if and only if $\Delta_{i}>0$. Conversely, it does not exist $A>0$ such that $F_{i^{*}}^{-1}(q-A) \geq$ $F_{i}^{-1}(q+A)$ if and only if $\Delta_{i}=0$. The last two statements are direct consequence of the definition of the gap $\Delta_{i}$ (see Definition 22.

\section{Corollary 1. Define}

$$
\begin{aligned}
Q_{R, F_{i}}(q) & \triangleq \inf \left\{x: F_{i}(x)>q\right\}, \\
L_{F_{i^{*}}}^{-}(q) & \triangleq \max _{x}\left\{F_{i^{*}}(x): F_{i^{*}}(x)<q\right\} .
\end{aligned}
$$

Assume that the best arm is unique,

$$
F_{i}^{-1}(q)<F_{i^{*}}^{-1}(q), \quad \forall i \in\{1,2 \ldots, K\} \backslash\left\{i^{*}\right\} .
$$

1) If the $C D F F_{i}(\cdot)$ is continuous at $Q_{R, F_{i}}(q)$ it is always true that $Q_{R, F_{i}}(q)<F_{i^{*}}^{-1}(q)$, and there exists $A>0$ such that $F_{i^{*}}^{-1}(q-A)>F_{i}^{-1}(q+A)$.

2) If the $C D F F_{i}(\cdot)$ is not continuous at $Q_{R, F_{i}}(q)$ then there are three sub-cases.

- $Q_{R, F_{i}}(q)<F_{i^{*}}^{-1}(q)$ : There exists $A>0$ such that

$$
F_{i^{*}}^{-1}(q-A)>F_{i}^{-1}(q+A) .
$$

The values of $A$ that satisfy (62) are

$$
A(\alpha, x)=\alpha \min \left\{F_{i}(x)-q, q-F_{i^{*}}(x)\right\},
$$

for any $\alpha \in(0,1)$ and $x \in\left[Q_{R, F_{i}}(q), F_{i^{*}}^{-1}(q)\right)$.

- $Q_{R, F_{i}}(q)=F_{i^{*}}^{-1}(q)$ : There does not exist $A>0$ such that $F_{i^{*}}^{-1}(q-A)>F_{i}^{-1}(q+A)$.

- If $F_{i^{*}}(\cdot)$ has a discontinuity at $F_{i^{*}}^{-1}(q)$ there exists $A>0$ such that

$$
F_{i^{*}}^{-1}(q-A)=F_{i}^{-1}(q+A) .
$$

The values of A that satisfy 64 are

$$
\begin{aligned}
A(\epsilon) & =\min \left\{F_{i}\left(Q_{R, F_{i}}(q)\right)-q, q-L_{F_{i^{*}}}^{-}(q)-\epsilon\right\} \\
& =\min \left\{F_{i}\left(F_{i^{*}}^{-1}(q)\right)-q, q-L_{F_{i^{*}}}^{-}(q)-\epsilon\right\}
\end{aligned}
$$

for any $\epsilon \in\left(0, q-L_{F_{i^{*}}}^{-}(q)\right]$.

- If $F_{i^{*}}(\cdot)$ is continuous at $F_{i^{*}}^{-1}(q)$ then there does not exist $A>0$ such that

$$
F_{i^{*}}^{-1}(q-A) \geq F_{i}^{-1}(q+A) .
$$

- $Q_{R, F_{i}}(q)>F_{i^{*}}^{-1}(q)$ : It does not exist $A>0$ such that

$$
F_{i^{*}}^{-1}(q-A) \geq F_{i}^{-1}(q+A) .
$$

A. Proof of the case $Q_{R, F_{i}}(q)<F_{i^{*}}^{-1}(q)$ in Corollary 1

We show that

$$
\Delta_{i} \triangleq \min \left\{F_{i}(x)-q, q-F_{i^{*}}(x)\right\}>0,
$$

for all $x \in\left[Q_{R, F_{i}}(q), F_{i^{*}}^{-1}(q)\right) \subset\left[F_{i}^{-1}(q), F_{i^{*}}^{-1}(q)\right]$ and

$$
F_{i^{*}}^{-1}\left(q-\alpha \Delta_{i}\right)>F_{i}^{-1}\left(q+\alpha \Delta_{i}\right),
$$

for all $x \in\left[Q_{R, F_{i}}(q), F_{i^{*}}^{-1}(q)\right)$ and $\alpha \in(0,1)$. To show 67) it is sufficient to find the minimum value $\tilde{x}$ in the interval $\left[F_{i}^{-1}(q), F_{i^{*}}^{-1}(q)\right]$ such that

$$
F_{i}(\tilde{x})-q>0 \text { and } q-F_{i^{*}}(\tilde{x})>0 .
$$

Notice that

$$
q-F_{i^{*}}(x)>0, \quad \forall x \in\left[F_{i}^{-1}(q), F_{i^{*}}^{-1}(q)\right),
$$

thus we have to find the minimum value $\tilde{x}$ in the interval $\left[F_{i}^{-1}(q), F_{i^{*}}^{-1}(q)\right)$ such that

$$
F_{i}(\tilde{x})-q>0 .
$$

By the assumption $Q_{R, F_{i}}(q)<F_{i^{*}}^{-1}(q)$ it follows that

$$
Q_{R, F_{i}}(q) \triangleq \inf \left\{x: F_{i}(x)>q\right\}<F_{i^{*}}^{-1}(q) .
$$

This implies that

$$
\tilde{x} \equiv Q_{R, F_{i}}(q) .
$$

Further

$$
F_{i}^{-1}(q) \leq Q_{R, F_{i}}(q)<F_{i^{*}}^{-1}(q),
$$

which implies that

$$
\Delta_{i} \triangleq \min \left\{F_{i}(x)-q, q-F_{i^{*}}(x)\right\}>0,
$$

for all $x \in\left[Q_{R, F_{i}}(q), F_{i^{*}}^{-1}(q)\right) \subset\left[F_{i}^{-1}(q), F_{i^{*}}^{-1}(q)\right]$.

For any $x \in\left[Q_{R, F_{i}}(q), F_{i^{*}}^{-1}(q)\right)$ and any $\alpha \in(0,1)$ it is true that

$$
F_{i^{*}}\left(F_{i^{*}}^{-1}\left(q-\alpha \Delta_{i}\right)\right) \geq q-\alpha \Delta_{i}>q-\Delta_{i} \geq F_{i^{*}}(x),
$$

where the last inequality comes from the definition of $\Delta_{i}$. As a consequence of 76

$$
\begin{aligned}
F_{i^{*}}\left(F_{i^{*}}^{-1}\left(q-\alpha \Delta_{i}\right)\right) & >F_{i^{*}}(x) \Longrightarrow \\
F_{i^{*}}^{-1}\left(q-\alpha \Delta_{i}\right) & >x
\end{aligned}
$$

because $F_{i^{*}}(\cdot)$ is increasing. Further, for any $x \in S$ and any $\alpha \in(0,1)$ it is true that

$$
x \geq F_{i}^{-1}\left(F_{i}(x)\right) \geq F_{i}^{-1}\left(q+\Delta_{i}\right) \geq F_{i}^{-1}\left(q+\alpha \Delta_{i}\right)
$$

because $F_{i}(x) \geq \Delta_{i}+q$ (that comes from the definition of the $\Delta_{i}$ ) and $F_{i}^{-1}(\cdot)$ is increasing. Now (77) and 78) give

$$
F_{i^{*}}^{-1}\left(q-\alpha \Delta_{i}\right)>F_{i}^{-1}\left(q+\alpha \Delta_{i}\right),
$$

for any $x \in\left[Q_{R, F_{i}}(q), F_{i^{*}}^{-1}(q)\right)$ and $\alpha \in(0,1)$. 


\section{B. Proof of the case $Q_{R, F_{i}}(q)=F_{i^{*}}^{-1}(q)$ in Corollary 1}

First we show that if $Q_{R, F_{i}}(q)=F_{i^{*}}^{-1}(q)$ and the best arm is unique $F_{i}^{-1}(q)<F_{i^{*}}^{-1}(q)$, then there does not exist $A>0$ such that

$$
F_{i^{*}}^{-1}(q-A)>F_{i}^{-1}(q+A) .
$$

We use contradiction to show that there does not exist $A>0$ such that $F_{i}^{-1}(q+A)=F_{i}^{-1}(q)$. Assume that for some $A>0$

$$
\begin{aligned}
F_{i}^{-1}(q) & =F_{i}^{-1}(q+A) \Longrightarrow \\
F_{i}\left(F_{i}^{-1}(q)\right) & =F_{i}\left(F_{i}^{-1}(q+A)\right) \geq q+A \Longrightarrow \\
F_{i}\left(F_{i}^{-1}(q)\right) & \geq q+A \Longrightarrow \\
F_{i}\left(F_{i}^{-1}(q)\right) & \geq F_{i}\left(Q_{R, F_{i}}(q)\right),
\end{aligned}
$$

where the last line cannot hold only as a strict inequality $F_{i}\left(F_{i}^{-1}(q)\right)>F_{i}\left(Q_{R, F_{i}}(q)\right)$ because of the monotonicity of $F_{i}(\cdot)$. Additionally, the definition $Q_{R, F_{i}}(q) \triangleq \inf \{x$ : $\left.F_{i}(x)>q\right\}$ gives that $F_{i}\left(F_{i}^{-1}(q)\right)=F_{i}\left(Q_{R, F_{i}}(q)\right)$ if and only if $Q_{R, F_{i}}(q)=F_{i}^{-1}(q)$. The latter does not hold because $Q_{R, F_{i}}(q)=F_{i^{*}}^{-1}(q)>F_{i}^{-1}(q)$. Combining the above we get the contradiction. As a consequence for every $A>0$ it is true that

$$
\begin{aligned}
& F_{i}^{-1}(q+A)>F_{i}^{-1}(q) \\
& \Longrightarrow F_{i}^{-1}(q+A) \geq Q_{R, F_{i}}(q) \\
& \Longrightarrow F_{i}^{-1}(q+A) \geq F_{i^{*}}^{-1}(q) \\
& \Longrightarrow F_{i}^{-1}(q+A) \geq F_{i^{*}}^{-1}(q-A) .
\end{aligned}
$$

The last line completes the statement of 80 , and the inequality $F_{i^{*}}^{-1}(q-A)>F_{i}^{-1}(q+A)$ holds only for $A=0$.

If the $\mathrm{CDF} F_{i^{*}}(\cdot)$ has a discontinuity at $F_{i^{*}}^{-1}(q)$ there exists $A>0$ such that

$$
F_{i^{*}}^{-1}(q-A)=F_{i}^{-1}(q+A) .
$$

Define $L_{F_{i^{*}}}^{-}(q) \triangleq \max _{x}\left\{F_{i^{*}}(x): F_{i^{*}}(x)<q\right\}$. and recall that $Q_{R, F_{i}}(q) \stackrel{=}{=} F_{i^{*}}^{-1}(q)$ then

$$
\begin{aligned}
A_{1} & \triangleq \sup \left\{A: F_{i}^{-1}(q+A)=Q_{R, F_{i}}(q)\right\}=F_{i}\left(Q_{R, F_{i}}(q)\right)-q \\
\mathcal{A}_{2} & \triangleq\left\{A: F_{i^{*}}^{-1}(q-A)=F_{i^{*}}^{-1}(q)\right\} \\
& =\left\{q-L_{F_{i^{*}}}^{-}(q)-\epsilon: \epsilon \in\left(0, q-L_{F_{i^{*}}}^{-}(q)\right]\right\} .
\end{aligned}
$$

For any $\epsilon \in\left(0, q-L_{F_{i^{*}}}^{-}(q)\right]$ define $A_{2}(\epsilon)=q-L_{F_{i^{*}}}^{-}(q)-\epsilon \epsilon$ $\mathcal{A}_{2}$, then the quantity

$$
A^{*}(\epsilon) \triangleq \min \left\{A_{1}, A_{2}(\epsilon)\right\}
$$

satisfies the condition

$$
F_{i^{*}}^{-1}\left(q-A^{*}(\epsilon)\right)=F_{i}^{-1}\left(q+A^{*}(\epsilon)\right) .
$$

On the other hand if $F_{i^{*}}(\cdot)$ is continuous at $F_{i^{*}}^{-1}(q)$ then for every $A>0$

$$
F_{i^{*}}^{-1}(q-A)<F_{i^{*}}^{-1}(q) .
$$

The latter combined with the inequality (82) give that for every $A>0$ it is true that $F_{i^{*}}^{-1}(q+A)>F_{i^{*}}^{-1}(q-A)$. As a consequence there does not exist $A>0$ such that

$$
F_{i^{*}}^{-1}(q-A) \geq F_{i}^{-1}(q+A) .
$$

${ }^{9}$ The level $q$ is not in the codomain of $F_{i}(\cdot)$

\section{Proof of the case $Q_{R, F_{i}}(q)>F_{i^{*}}^{-1}(q)$ in Corollary 1}

We use contradiction to show that there does not exist $A>0$ such that $F_{i}^{-1}(q+A)=F_{i}^{-1}(q)$. Assume that for some $A>0$

$$
\begin{aligned}
F_{i}^{-1}(q) & =F_{i}^{-1}(q+A) \Longrightarrow \\
F_{i}\left(F_{i}^{-1}(q)\right) & =F_{i}\left(F_{i}^{-1}(q+A)\right) \geq q+A \Longrightarrow \\
F_{i}\left(F_{i}^{-1}(q)\right) & \geq q+A \Longrightarrow \\
F_{i}\left(F_{i}^{-1}(q)\right) & \geq F_{i}\left(Q_{R, F_{i}}(q)\right),
\end{aligned}
$$

where the last line cannot hold only as a strict inequality $F_{i}\left(F_{i}^{-1}(q)\right)>F_{i}\left(Q_{R, F_{i}}(q)\right)$ because of the monotonicity of $F_{i}(\cdot)$. Additionally, the definition $Q_{R, F_{i}}(q) \triangleq \inf \{x$ : $\left.F_{i}(x)>q\right\}$ gives that $F_{i}\left(F_{i}^{-1}(q)\right)=F_{i}\left(Q_{R, F_{i}}(q)\right)$ if and only if $Q_{R, F_{i}}(q)=F_{i}^{-1}(q)^{10}$ The latter does not hold because $Q_{R, F_{i}}(q)=F_{i^{*}}^{-1}(q)>F_{i}^{-1}(q)$. Combining the above we get the contradiction. For every $A>0$ it is true that

$$
\begin{aligned}
& F_{i}^{-1}(q+A)>F_{i}^{-1}(q) \\
& \quad \Longrightarrow F_{i}^{-1}(q+A) \geq Q_{R, F_{i}}(q) \\
& \Longrightarrow F_{i}^{-1}(q+A)>F_{i^{*}}^{-1}(q) \\
& \Longrightarrow F_{i}^{-1}(q+A)>F_{i^{*}}^{-1}(q-A) .
\end{aligned}
$$

As a consequence, there does not exist $A>0$ such that

$$
F_{i^{*}}^{-1}(q-A) \geq F_{i}^{-1}(q+A) .
$$

\section{REFERENCES}

[1] T. Lattimore and C. Szepesvári, Bandit Algorithms. Cambridge, UK Cambridge University Press, 2020.

[2] O. Madani, D. J. Lizotte, and R. Greiner, "The budgeted multi-armed bandit problem," in International Conference on Computational Learning Theory, ser. Lecture Notes in Computer Science, J. Shawe-Taylor and Y. Singer, Eds., vol. 2130. Berlin, Heidelberg: Springer, 2004, pp. 643645. [Online]. Available: https://doi.org/10.1007/978-3-540-27819-1_46

[3] S. Bubeck, R. Munos, and G. Stoltz, "Pure exploration in multi-armed bandits problems," in Algorithmic Learning Theory, R. Gavaldà, G. Lugosi, T. Zeugmann, and S. Zilles, Eds. Berlin, Heidelberg: Springer Berlin Heidelberg, 2009, pp. 23-37. [Online]. Available: https://doi.org/10.1007/978-3-642-04414-4_7

[4] J. Y. Yu and E. Nikolova, "Sample complexity of risk-averse bandit-arm selection," in Twenty-Third International Joint Conference on Artificial Intelligence, 2013. [Online]. Available: https://www.aaai.org/ocs/index php/IJCAI/IJCAI13/paper/view/6194/7094

[5] B. Szörényi, R. Busa-Fekete, P. Weng, and E. Hüllermeier, "Qualitative multi-armed bandits: A quantile-based approach," in Proceedings of the 32nd International Conference on Machine Learning, ser. Proceedings of Machine Learning Research, F. Bach and D. Blei, Eds., vol. 37. Lille, France: PMLR, 07-09 Jul 2015, pp. 1660-1668. [Online]. Available: https://proceedings.mlr.press/v37/szorenyi15.html

[6] A. Ruszczyński and A. Shapiro, "Optimization of convex risk functions," Mathematics of operations research, vol. 31, no. 3, pp. 433-452, 2006.

[7] A. Sani, A. Lazaric, and R. Munos, "Risk-aversion in multi-armed bandits," in Advances in Neural Information Processing Systems 25, F. Pereira, C. J. C. Burges, L. Bottou, and K. Q. Weinberger, Eds. Curran Associates, Inc., 2012, pp. 3275-3283. [Online]. Available: https: //papers.nips.cc/paper/4753-risk-aversion-in-multi-armed-bandits.pdf

[8] A. Shapiro, "Minimax and risk averse multistage stochastic programming," European Journal of Operational Research, vol. 219, no. 3, pp. 719-726, 2012.

[9] A. Shapiro, D. Dentcheva, and A. Ruszczyński, Lectures on Stochastic Programming: Modeling and Theory, 2nd ed. Society for Industrial and Applied Mathematics, 2014.

[10] A. Tamar, Y. Chow, M. Ghavamzadeh, and S. Mannor, "Sequential decision making with coherent risk," IEEE Transactions on Automatic Control, vol. 62, no. 7, pp. 3323-3338, July 2017. [Online]. Available: https://doi.org/10.1109/TAC.2016.2644871

${ }^{10}$ The level $q$ is not in the codomain of $F_{i}(\cdot)$ 
[11] D. R. Jiang and W. B. Powell, "Risk-averse approximate dynamic programming with quantile-based risk measures," Mathematics of Operations Research, vol. 43, no. 2, pp. 554-579, nov 2018. [Online] Available: https://pubsonline.informs.org/doi/10.1287/moor.2017.0872

[12] W. Huang and W. B. Haskell, "Risk-aware Q-learning for Markov decision processes," in 2017 IEEE 56th Annual Conference on Decision and Control, CDC 2017, vol. 2018-Janua. IEEE, December 2018, pp. 4928-4933. [Online]. Available: https://doi.org/10.1109/CDC.2017 8264388

[13] D. S. Kalogerias and W. B. Powell, "Recursive optimization of convex risk measures: Mean-semideviation models," ArXiV, Tech Rep. arXiv:1804.00636 [math.OC], April 2018. [Online]. Available: https://arxiv.org/abs/1804.00636

[14] C. A. Vitt, D. Dentcheva, and H. Xiong, "Risk-Averse Classification," Annals of Operations Research, aug 2019.

[15] D. S. Kalogerias and W. B. Powell, "Zeroth-order algorithms for risk-aware learning," ArXiV, Tech. Rep. arXiv:1912.09484 [math.OC], December 2019. [Online]. Available: https://arxiv.org/abs/1912.09484

[16] A. Kagrecha, J. Nair, and K. Jagannathan, "Distribution oblivious, risk-aware algorithms for multi-armed bandits with unbounded rewards," in Advances in Neural Information Processing Systems, H. Wallach, H. Larochelle, A. Beygelzimer, F. d'Alché Buc, E. Fox, and R. Garnett, Eds., vol. 32. Curran Associates, Inc., 2019. [Online]. Available: https://proceedings.neurips.cc/paper/2019/file/ da54dd5a0398011cdfa50d559c2c0ef8-Paper.pdf

[17] A. R. Cardoso and H. Xu, "Risk-averse stochastic convex bandit," in Proceedings of Machine Learning Research, ser. Proceedings of Machine Learning Research, K. Chaudhuri and M. Sugiyama, Eds., vol. 89. PMLR, 16-18 Apr 2019, pp. 39-47. [Online]. Available: https://proceedings.mlr.press/v89/cardoso19a.html

[18] S.-K. Kim, R. Thakker, and A. Agha-Mohammadi, "Bi-directional value learning for risk-aware planning under uncertainty," IEEE Robotics and Automation Letters, vol. 4, no. 3, pp. 2493-2500, jul 2019. [Online]. Available: https://doi.org/10.1109/LRA.2019.2903259

[19] L. Zhou and P. Tokekar, "An approximation algorithm for risk-averse submodular optimization," in Springer Proceedings in Advanced Robotics, vol. 14. Springer, Cham, December 2020, pp. 144-159. [Online]. Available: https://doi.org/10.1007/978-3-030-44051-0_9

[20] A. A. Gaivoronski and G. Pflug, "Value-at-risk in portfolio optimization: properties and computational approach," Journal of Risk, vol. 7, no. 2, pp 1-31, 2005. [Online]. Available: https://doi.org/10.21314/JOR.2005.106

[21] J. Dean and L. A. Barroso, "The tail at scale," Communications of the ACM, vol. 56, no. 2, pp. 74-80, 2013. [Online]. Available: https://doi.org/10.1145/2408776.2408794

[22] C. Dwork, F. McSherry, K. Nissim, and A. Smith, "Calibrating noise to sensitivity in private data analysis," in Theory of Cryptography, ser. Lecture Notes in Computer Science. Springer, Berlin, Heidelberg, 2006 pp. 265-284. [Online]. Available: https://doi.org/10.1007/11681878_14

[23] Y. David and N. Shimkin, "Pure exploration for max-quantile bandits," in Joint European Conference on Machine Learning and Knowledge Discovery in Databases. Springer, 2016, pp. 556-571.

[24] S. Howard and A. Ramdas, "Sequential estimation of quantiles with applications to A/B-testing and best-arm identification," ArXiV, Tech. Rep. arXiv:1906.09712 [math.ST], 2019. [Online]. Available: https://arxiv.org/abs/1906.09712

[25] A. Beimel, K. Nissim, and U. Stemmer, "Characterizing the sample complexity of private learners," in Proceedings of the 4th Conference on Innovations in Theoretical Computer Science, ser. ITCS '13. New York, NY, USA: Association for Computing Machinery, 2013, p. 97-110 [Online]. Available: https://doi.org/10.1145/2422436.2422450

[26] V. Feldman and D. Xiao, "Sample complexity bounds on differentially private learning via communication complexity," in Proceedings of The 27th Conference on Learning Theory, COLT 2014, Barcelona, Spain, June 13-15, 2014, ser. JMLR Workshop and Conference Proceedings, M. Balcan, V. Feldman, and C. Szepesvári, Eds., vol. 35. JMLR.org, 2014, pp. 1000-1019.

[27] M. Bun, K. Nissim, U. Stemmer, and S. Vadhan, "Differentially private release and learning of threshold functions," in 2015 IEEE 56th Annual Symposium on Foundations of Computer Science, 2015, pp. 634-649. [Online]. Available: https://doi.org/10.1109/FOCS.2015.45

[28] E. Even-Dar, S. Mannor, and Y. Mansour, "PAC bounds for multi-armed bandit and markov decision processes," in International Conference on Computational Learning Theory, ser. Lecture Notes in Artificial Intelligence, J. Kivinen and R. H. Sloan, Eds., vol. 2375. Springer, 2002, pp. 255-270. [Online]. Available: https://doi.org/10.1007/3-540-45435-7_18
[29] S. Kalyanakrishnan, A. Tewari, P. Auer, and P. Stone, "PAC subset selection in stochastic multi-armed bandits." in Proceedings of the 2012 International Conference on Machine Learning (ICML), vol. 12, 2012, pp. 655-662.

[30] V. Gabillon, M. Ghavamzadeh, and A. Lazaric, "Best arm identification: A unified approach to fixed budget and fixed confidence," in Advances in Neural Information Processing Systems, F. Pereira, C. J. C. Burges, L. Bottou, and K. Q. Weinberger, Eds., vol. 25. Curran Associates, Inc., 2012. [Online]. Available: https://proceedings.neurips.cc/paper/2012/file/ 8b0d268963dd0cfb808aac48a549829f-Paper.pdf

[31] Z. Karnin, T. Koren, and O. Somekh, "Almost optimal exploration in multi-armed bandits," in Proceedings of the 30th International Conference on Machine Learning, ser. Proceedings of Machine Learning Research, S. Dasgupta and D. McAllester, Eds., vol. 28, no. 3. Atlanta, Georgia, USA: PMLR, 17-19 Jun 2013, pp. 1238-1246. [Online]. Available: http://proceedings.mlr.press/v28/karnin13.html

[32] K. Jamieson, M. Malloy, R. Nowak, and S. Bubeck, "On finding the largest mean among many," ArXiV, Tech. Rep. arXiv:1306.3917 [stat.ML], 2013. [Online]. Available: https://arxiv.org/abs/1306.3917

[33] _ "lil' UCB : An optimal exploration algorithm for multi-armed bandits," in Proceedings of The 27th Conference on Learning Theory, ser. Proceedings of Machine Learning Research, M. F. Balcan, V. Feldman, and C. Szepesvári, Eds., vol. 35. Barcelona, Spain: PMLR, 13-15 Jun 2014, pp. 423-439. [Online]. Available: http://proceedings.mlr.press/v35/jamieson14.html

[34] S. Mannor and J. N. Tsitsiklis, "The sample complexity of exploration in the multi-armed bandit problem," Journal of Machine Learning Research, vol. 5, pp. 623-648, June 2004. [Online]. Available: https://www.jmlr.org/papers/v5/mannor04b.html

[35] M. Anthony and P. L. Bartlett, Neural Network Learning: Theoretical Foundations. Cambridge, UK: Cambridge University Press, 2009.

[36] A. N. Burnetas and M. N. Katehakis, "Optimal adaptive policies for sequential allocation problems," Advances in Applied Mathematics, vol. 17, no. 2, pp. 122-142, 1996. [Online]. Available: https: //doi.org/10.1006/aama.1996.0007

[37] L. Chen and J. Li, "On the optimal sample complexity for best arm identification," ArXiV, Tech. Rep. arXiv:1511.03774 [cs.LG], August 2016. [Online]. Available: https://arxiv.org/abs/1511.03774

[38] E. Kaufmann, O. Cappe, and A. Garivier, "On the complexity of best-arm identification in multi-armed bandit models," The Journal of Machine Learning Research, vol. 17, no. 1, pp. 1-42, 2016. [Online]. Available: https://jmlr.csail.mit.edu/papers/v17/kaufman16a.html

[39] A. Garivier and E. Kaufmann, "Optimal best arm identification with fixed confidence," in 29th Annual Conference on Learning Theory, ser. Proceedings of Machine Learning Research, V. Feldman, A. Rakhlin, and O. Shamir, Eds., vol. 49. Columbia University, New York, New York, USA: PMLR, 23-26 Jun 2016, pp. 998-1027. [Online]. Available: http://proceedings.mlr.press/v49/garivier16a.html

[40] O. Cappé, A. Garivier, O.-A. Maillard, R. Munos, and G. Stoltz, "Kullback-Leibler upper confidence bounds for optimal sequential allocation," The Annals of Statistics, vol. 41, no. 3, pp. 1516-1541, 2013. [Online]. Available: https://doi.org/10.1214/13-AOS1119

[41] K. Jamieson and A. Talwalkar, "Non-stochastic best arm identification and hyperparameter optimization," in Proceedings of the 19th International Conference on Artificial Intelligence and Statistics, ser Proceedings of Machine Learning Research, A. Gretton and C. C. Robert, Eds., vol. 51. Cadiz, Spain: PMLR, 09-11 May 2016, pp. 240-248. [Online]. Available: http://proceedings.mlr.press/v51/jamieson16.html

[42] L. Li, K. Jamieson, G. DeSalvo, A. Rostamizadeh, and A. Talwalkar, "Hy perband: A novel bandit-based approach to hyperparameter optimization," J. Mach. Learn. Res., vol. 18, no. 1, p. 6765-6816, Jan. 2017.

[43] R. Allesiardo and R. Feraud, "Selection of learning experts," in 2017 International Joint Conference on Neural Networks (IJCNN), 2017, pp. 1005-1010. [Online]. Available: https://doi.org/10.1109/IJCNN.2017. 7965962

[44] R. Allesiardo, R. Féraud, and O.-A. Maillard, "The non-stationary stochastic multi-armed bandit problem," International Journal of Data Science and Analytics, vol. 3, no. 4, pp. 267-283, 2017. [Online]. Available: https://doi.org/10.1007/s41060-017-0050-5

[45] S. Bubeck and N. Cesa-Bianchi, "Regret analysis of stochastic and nonstochastic multi-armed bandit problems," Foundations and Trends $®$ in Machine Learning, vol. 5, no. 1, pp. 1-122, 2012.

[46] S. Bubeck, N. Cesa-Bianchi, and G. Lugosi, "Bandits with heavy tail," IEEE Transactions on Information Theory, vol. 59, no. 11, pp. 7711-7717, 2013. [Online]. Available: https://doi.org/10.1109/TIT.2013.2277869

[47] B. Li, T. Chen, and G. B. Giannakis, "Bandit online learning with unknown delays," in Proceedings of the Twenty-Second International 
Conference on Artificial Intelligence and Statistics, ser. Proceedings of Machine Learning Research, K. Chaudhuri and M. Sugiyama, Eds., vol. 89. PMLR, 16-18 Apr 2019, pp. 993-1002. [Online]. Available: http://proceedings.mlr.press/v89/li19d.html

[48] Q. Berthet and V. Perchet, "Fast rates for bandit optimization with upper-confidence Frank-Wolfe," in Advances in Neural Information Processing Systems, I. Guyon, U. V. Luxburg, S. Bengio, H. Wallach, R. Fergus, S. Vishwanathan, and R. Garnett, Eds., vol. 30. Curran Associates, Inc., 2017. [Online]. Available: https://proceedings.neurips cc/paper/2017/file/dc960c46c38bd16e953d97cdeefdbc68-Paper.pdf

[49] V. P. Boda and P. L.A., "Correlated bandits or: How to minimize mean-squared error online," in Proceedings of the 36th International Conference on Machine Learning, ser. Proceedings of Machine Learning Research, K. Chaudhuri and R. Salakhutdinov, Eds., vol. 97. PMLR, 09-15 Jun 2019, pp. 686-694. [Online]. Available: http://proceedings.mlr.press/v97/boda19a.html

[50] O.-A. Maillard, "Robust risk-averse stochastic multi-armed bandits," in Algorithmic Learning Theory, S. Jain, R. Munos, F. Stephan, and T. Zeugmann, Eds. Berlin, Heidelberg: Springer Berlin Heidelberg, 2013, pp. 218-233. [Online]. Available: https://dx.doi.org/10.1007/ 978-3-642-40935-6_16

[51] E. Even-Dar, M. Kearns, and J. Wortman, "Risk-sensitive online learning," in Algorithmic Learning Theory, J. L. Balcázar, P. M. Long, and F. Stephan, Eds. Berlin, Heidelberg: Springer Berlin Heidelberg, 2006, pp. 199-213. [Online]. Available: https://dx.doi.org/10.1007/ $11894841 \_18$

[52] S. Vakili and Q. Zhao, "Risk-averse multi-armed bandit problems under mean-variance measure," IEEE Journal of Selected Topics in Signal Processing, vol. 10, no. 6, pp. 1093-1111, 2016. [Online]. Available: https://doi.org/10.1109/JSTSP.2016.2592622

[53] A. Cassel, S. Mannor, and A. Zeevi, "A general approach to multi-armed bandits under risk criteria," in Proceedings of the 31st Conference On Learning Theory, ser. Proceedings of Machine Learning Research, S. Bubeck, V. Perchet, and P. Rigollet, Eds., vol. 75. PMLR, 06-09 Jul 2018, pp. 1295-1306. [Online]. Available: http://proceedings.mlr.press/v75/cassel18a.html

[54] Y. Wang and F. Gao, "Deviation inequalities for an estimator of the conditional value-at-risk," Operations Research Letters, vol. 38, no. 3, pp. 236-239, 2010. [Online]. Available: https: //doi.org/10.1016/j.orl.2009.11.008

[55] R. K. Kolla, L. Prashanth, S. P. Bhat, and K. Jagannathan, "Concentration bounds for empirical conditional value-at-risk: The unbounded case," Operations Research Letters, vol. 47, no. 1, pp. 16-20, 2019. [Online]. Available: https://doi.org/10.1016/j.orl.2018.11.005

[56] S. P. Bhat and P. L.A., "Concentration of risk measures: A Wasserstein distance approach," in Advances in Neural Information Processing Systems, H. Wallach, H. Larochelle, A. Beygelzimer, F. d'Alché Buc, E. Fox, and R. Garnett, Eds., vol. 32. Curran Associates, Inc., 2019. [Online]. Available: https://proceedings.neurips.cc/paper/2019/file/ 091bc5440296cc0e41dd60ce22fbaf88-Paper.pdf

[57] L. Torossian, A. Garivier, and V. Picheny, " $\mathcal{X}$-armed bandits: Optimizing quantiles, CVaR and other risks," in Proceedings of The Eleventh Asian Conference on Machine Learning, ser. Proceedings of Machine Learning Research, W. S. Lee and T. Suzuki, Eds., vol. 101. Nagoya Japan: PMLR, 17-19 Nov 2019, pp. 252-267. [Online]. Available: https://proceedings.mlr.press/v101/torossian19a.html

[58] J. Altschuler, V.-E. Brunel, and A. Malek, "Best arm identification for contaminated bandits," Journal of Machine Learning Research, vol. 20, no. 91, pp. 1-39, 2019. [Online]. Available: https: //www.jmlr.org/papers/v20/18-395.html

[59] K. Nissim, S. Raskhodnikova, and A. Smith, "Smooth sensitivity and sampling in private data analysis," in Proceedings of the Thirty-Ninth Annual ACM Symposium on Theory of Computing, ser. STOC '07. New York, NY, USA: Association for Computing Machinery, 2007, p. 75-84. [Online]. Available: https://doi.org/10.1145/1250790.1250803

[60] K. Chaudhuri and D. Hsu, "Sample complexity bounds for differentially private learning," in Proceedings of the 24th Annual Conference on Learning Theory, ser. Proceedings of Machine Learning Research, S. M. Kakade and U. von Luxburg, Eds., vol. 19. Budapest, Hungary: PMLR, 09-11 Jun 2011, pp. 155-186. [Online]. Available: http://proceedings.mlr.press/v19/chaudhuri11a.html

[61] A. Beimel, K. Nissim, and U. Stemmer, "Private learning and sanitization: Pure vs. approximate differential privacy," in Approximation, Randomization, and Combinatorial Optimization. Algorithms and Techniques, P. Raghavendra, S. Raskhodnikova, K. Jansen, and J. D. P. Rolim, Eds. Berlin, Heidelberg: Springer Berlin Heidelberg,
2013, pp. 363-378. [Online]. Available: https://doi.org/10.1007/ 978-3-642-40328-6_26

[62] N. Alon, R. Livni, M. Malliaris, and S. Moran, "Private PAC learning implies finite Littlestone dimension," in Proceedings of the 51st Annual ACM SIGACT Symposium on Theory of Computing, ser. STOC 2019. New York, NY, USA: Association for Computing Machinery, 2019, p. 852-860. [Online]. Available: https://doi.org/10.1145/3313276.3316312

[63] H. Kaplan, K. Ligett, Y. Mansour, M. Naor, and U. Stemmer, "Privately learning thresholds: Closing the exponential gap," in Proceedings of Thirty Third Conference on Learning Theory, ser. Proceedings of Machine Learning Research, J. Abernethy and S. Agarwal, Eds., vol. 125. PMLR, 09-12 Jul 2020, pp. 2263-2285. [Online]. Available: http://proceedings.mlr.press/v125/kaplan20a.html

[64] N. Mishra and A. Thakurta, "(Nearly) optimal differentially private stochastic multi-arm bandits," in Proceedings of the Thirty-First Conference on Uncertainty in Artificial Intelligence. AUAI Press, 2015, pp. 592-601. [Online]. Available: http://auai.org/uai2015/proceedings/ papers/58.pdf

[65] P. Auer, N. Cesa-Bianchi, and P. Fischer, "Finite-time analysis of the multiarmed bandit problem," Machine Learning, vol. 47, no. 2-3, pp. 235 256, 2002. [Online]. Available: https://doi.org/10.1023/A:1013689704352

[66] T.-H. H. Chan, E. Shi, and D. Song, "Private and continual release of statistics," ACM Trans. Inf. Syst. Secur, vol. 14, no. 3, Nov. 2011. [Online]. Available: https://doi.org/10.1145/2043621.2043626

[67] C. Dwork, M. Naor, T. Pitassi, and G. N. Rothblum, "Differential privacy under continual observation," in Proceedings of the Forty-Second ACM Symposium on Theory of Computing, ser. STOC '10. New York, NY, USA: Association for Computing Machinery, 2010, p. 715-724. [Online]. Available: https://doi.org/10.1145/1806689.1806787

[68] R. Shariff and O. Sheffet, "Differentially private contextual linear bandits," in Advances in Neural Information Processing Systems, S. Bengio, H. Wallach, H. Larochelle, K. Grauman, N. CesaBianchi, and R. Garnett, Eds., vol. 31. Curran Associates, Inc., 2018. [Online]. Available: https://proceedings.neurips.cc/paper/2018/file/ a1d7311f2a312426d710e1c617fcbc8c-Paper.pdf

[69] T. Sajed and O. Sheffet, "An optimal private stochastic-MAB algorithm based on optimal private stopping rule," in Proceedings of the 36th International Conference on Machine Learning, ser. Proceedings of Machine Learning Research, K. Chaudhuri and R. Salakhutdinov, Eds. vol. 97. PMLR, 09-15 Jun 2019, pp. 5579-5588. [Online]. Available: http://proceedings.mlr.press/v97/sajed19a.html

[70] C. Dwork and A. Roth, "The algorithmic foundations of differential privacy," Foundations and Trends ${ }^{\circledR}$ in Theoretical Computer Science, vol. 9, no. 3-4, pp. 211-407, Aug. 2014.

[71] C. Dwork, G. Rothblum, and S. Vadhan, "Boosting and differential privacy," in 2010 51st Annual IEEE Symposium on Foundations of Computer Science (FOCS), Las Vegas, NV, October 2010, pp. 51-60. [Online]. Available: https://doi.org/10.1109/FOCS.2010.12

[72] P. Kairouz, S. Oh, and P. Viswanath, "The composition theorem for differential privacy," IEEE Transactions on Information Theory, vol. 63, no. 6, June 2017. [Online]. Available: https://doi.org/10.1109/TIT.2017. 2685505 